\title{
Avifauna da Estação Ecológica do Rio Acre, estado do Acre, na fronteira Brasil/Peru: composição, distribuição ecológica e registros relevantes Avifauna of the Estação Ecológica do Rio Acre, state of Acre, on the Brazil/Peru border: composition, ecological distribution, and noteworthy records
}

Alexandre Aleixo'

Edson Guilherme

Resumo: Um levantamento da avifauna foi executado na Estação Ecológica do Rio Acre (ESEC Rio Acre), uma unidade de conservação remota situada na região sul do estado do Acre, na fronteira com o Peru, com a finalidade de subsidiar o seu plano de manejo. Os trabalhos de campo foram realizados nas épocas seca (agosto de 2005) e chuvosa (fevereiro de 2006), com a realização de levantamentos qualitativos (observações e gravações de vocalizações) e quantitativos, por meio da captura com redes de neblina (mist-nets). Após o acúmulo de 180 horas de observações qualitativas e cerca de 2.500 horas.rede de capturas distribuídos em 26 dias de amostragem, registrou-se 365 espécies de aves. Vários dos registros obtidos na ESEC Rio Acre são aqui discutidos em detalhes em função da sua relevância biogeográfica e para a conservação. Os resultados gerais obtidos, aliados ao registro de grandes populações de espécies de aves com alto valor cinegético, indicam que a ESEC Rio Acre encontra-se ainda em ótimo estado de conservação. Infelizmente, essa situação pode mudar rapidamente caso a expansão madeireira, atualmente em curso no lado Peruano da fronteira (em área limítrofe à unidade), seja direcionada ilegalmente para o território brasileiro.

Palavras-chave: Amazônia. Aves. Biogeografia. Acre. Conservação. Extensão de distribuição.

Abstract: We carried out an ornithological inventory at the Estação Ecológica do Rio Acre (ESEC Rio Acre), a remote conservation unit in southern Acre, on the Brazil/Peru border, to contribute data for the unit's management plan. Surveys took place during two separate expeditions in the dry (August 2005) and the wet season (February 2006), and included qualitative (observations, tape-recording) as well as quantitative (mist-netting) sampling techniques. We accumulated 180 hours of qualitative observations and ca. 2,500 mist-net.hours during 26 days, recording 365 species of birds. Several noteworthy records are discussed in detail here due to their relevance for Amazonian biogeography and conservation. The general results obtained, including the observation of large populations of bird species that are highly valued for hunting, indicate that ESEC Rio Acre is still very well preserved. Unfortunately, this can easily change if logging, which is currently taking place on the Peruvian side of the border adjacent to ESEC Rio Acre, crosses the border illegally into Brazil.

Keywords: Amazonia. Birds. Biogeography. Acre. Conservation. Range extension.

Museu Paraense Emílio Goeldi. Coordenação de Zoologia. Belém, Pará, Brasil (aleixo@museu-goeldi.br).

" Universidade Federal do Acre. Centro de Ciências Biológicas e da Natureza. Laboratório de Paleontologia. Rio Branco, Acre, Brasil (guilherme@ufac.br).

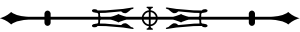




\section{INTRODUÇÃO}

O estado do Acre é considerado uma das áreas de maior diversidade ornitológica no planeta, com a ocorrência simpátrica de mais de 600 espécies de aves em algumas localidades (Pinto \& Camargo, 1954; Novaes, 1957, 1958; Whittaker \& Oren, 1999; Guilherme, 2001; Whittaker et al., 2002; Rasmussen et al., 2005). Apesar desta grande diversidade já documentada, um número relativamente pequeno de localidades do Acre foi amostrado por ornitólogos nas últimas décadas (Oren \& Albuquerque, 1991), sendo que estas, na sua maioria, estão localizadas ao longo do rio Juruá, na porção oeste do estado (Novaes, 1957, 1958; Whittaker \& Oren, 1999; Whittaker et al., 2002). Na intenção de mudar este cenário, o Acre foi alvo de diversas expedições ornitológicas nos últimos cinco anos, tanto na sua porção leste quanto na central (Guilherme, 2007; Aleixo \& Guilherme, 2008; Guilherme \& Dantas, 2008; Guilherme \& Santos, 2009). Este incremento de estudos no estado do Acre tem confirmado a alta diversidade avifaunística local e contribuído significativamente para a diminuição das lacunas de conhecimento da biodiversidade no sudoeste da Amazônia brasileira.

A região do alto rio Acre, situada na porção sudeste do estado e adjacente aos territórios da Bolívia e do Peru, constitui uma das menos conhecidas no Brasil do ponto de vista ornitológico, havendo em território brasileiro apenas um levantamento preliminar prévio realizado nas proximidades do município de Assis Brasil (Guilherme, 2004). Localidades da mesma região amostradas na Bolívia e no Peru revelaram a presença de uma avifauna riquíssima, com várias espécies endêmicas da área de endemismo Inambari (Gyldenstolpe, 1945; Haffer, 1978; Parker, 1982; Terborgh et al., 1984, 1990; Cracraft, 1985; Parker \& Remsen, 1987; Parker et al., 1994; Servat, 1996; Tobias \& Seddon, 2007), sendo algumas delas ainda não registradas em território brasileiro (CBRO, 2009).

No contexto do projeto para a elaboração do plano de manejo da Estação Ecológica do Rio Acre (denominada ESEC Rio Acre daqui em diante), foram executados os primeiros levantamentos detalhados de avifauna do lado brasileiro da região do alto rio Acre. A seguir, os resultados obtidos são apresentados e as suas implicações biogeográficas e para a conservação são discutidas.

\section{MATERIAL E MÉTODOS}

A ESEC Rio Acre é uma unidade de conservação de proteção integral, com 77.500 ha, situada no município de Assis Brasil (sudeste do estado do Acre), na fronteira com o departamento peruano de Madre de Dios (ISA, 2009; Figura 1). A ESEC Rio Acre faz divisa com duas terras indígenas brasileiras: Mamoadate, ao norte, e Cabeceira do rio Acre, ao leste; o limite sul é delimitado pelo rio Acre na divisa Brasil/Peru. Entre 13 e 24 de agosto de 2005 e 3 e 16 de fevereiro de 2006, realizamos um inventário de avifauna na ESEC Rio Acre ao longo de transectos distribuídos em 13 localidades distintas, compreendendo as principais fitofisionomias e ambientes da unidade, onde predominam formações de floresta aluvial aberta, com manchas de bambus e/ou palmeiras.

O levantamento qualitativo de avifauna consistiu em caminhadas diurnas e noturnas através dos três ambientes e fitofisionomias distintos na área de estudo, com o objetivo de registrar as aves presentes a partir de contatos visuais e auditivos. $\bigcirc$ esforço de amostragem total deste levantamento foi de $180 \mathrm{~h}$, concentradas no período diurno e assim distribuídas: 96 h durante a estação seca (expedição de agosto de 2005) e 84 h durante a estação chuvosa (expedição de fevereiro de 2006). O esforço de amostragem noturno foi de apenas três horas em cada estação, totalizando seis horas. Durante este levantamento, diversas espécies de aves observadas foram documentadas com a gravação em fita cassete de suas vocalizações por um gravador Sony ${ }^{\circledR}$ TCM-5000 acoplado a um microfone direcional Sennheiser ${ }^{\circledR}$ ME-88. As gravações encontram-se depositadas no arquivo sonoro da Coleção Ornitológica do Museu Paraense Emílio Goeldi (MPEG), em Belém, Pará. Foram utilizadas redes de neblina de $12 \times 2 \mathrm{~m}$ para a captura de aves com o objetivo de complementar o levantamento qualitativo descrito acima. A cada dia de 


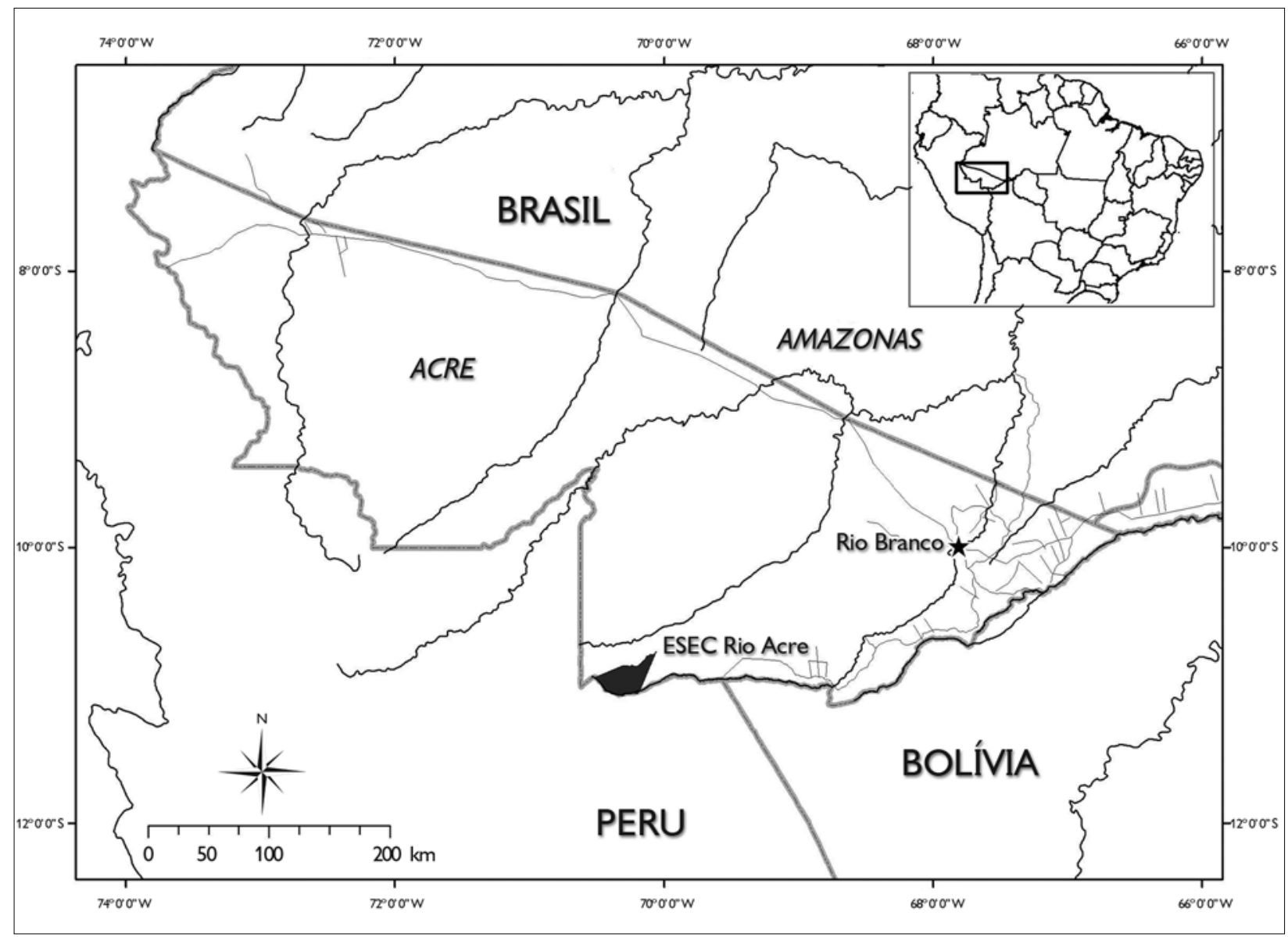

Figura 1. Mapa com a localização da ESEC Rio Acre.

amostragem, redes instaladas nas proximidades dos mesmos transectos percorridos durante o levantamento qualitativo permaneceram abertas durante a manhã por um período de pelo menos seis horas (geralmente entre 6 e 12 h). Um total de 20 redes de neblina foi utilizado ao longo de dez dias em agosto e 11 dias em fevereiro, permitindo o acúmulo de um esforço total de amostragem de aproximadamente 2.500 h.rede, distribuído de maneira relativamente equitativa entre as estações seca e chuvosa.

Foram coletados espécimes-testemunho de várias espécies de aves registradas na ESEC Rio Acre, notadamente aquelas de difícil identificação e de ocorrência previamente não documentada no Brasil. Essas coletas foram realizadas com o auxílio das redes de neblina e de uma espingarda, sendo devidamente autorizadas pelo Instituto Brasileiro do Meio Ambiente e dos Recursos Naturais Renováveis (IBAMA) (Licença 029/2005-CGFAU). Os espécimes coletados foram preparados diretamente no campo e posteriormente depositados na Coleção Ornitológica do Museu Paraense Emílio Goeldi (MPEG), em Belém, Pará.

\section{RESULTADOS E DISCUSSÃO}

\section{RIQUEZA DE ESPÉCIES, COMPOSIÇÃO E} DISTRIBUIÇÃO ECOLÓGICA DA AVIFAUNA

Durante os levantamentos de campo na ESEC Rio Acre, foram registradas 365 espécies de aves, cuja nomenclatura, fitofisionomias predominantes em que foram observadas e 
tipo de documentação obtido para algumas delas (gravação de vocalizações e/ou coleta de espécimes-testemunho) são apresentados em conjunto no Apêndice. Das espécies constantes no Apêndice, apenas três - Cochlearius cochlearius (Linnaeus, 1766), Conopophaga peruviana (Des Murs, 1856) e Ramphocelus nigrogularis (Spix, 1825) - não foram registradas dentro dos limites da ESEC Rio Acre durante os inventários. Estas espécies foram observadas em 2002 por Edson Guilherme (EG) em floresta aluvial (margem esquerda do rio Acre) dentro da Terra Indígena 'Cabeceira do Rio Acre', poucos quilômetros à jusante do limite com a ESEC Rio Acre.

A curva cumulativa de espécies de aves registradas pelo esforço de amostragem qualitativa não se estabilizou completamente (Figura 2). Entretanto, a diminuição na taxa de acúmulo de espécies observadas já a partir de $125 \mathrm{~h}$ acumuladas de observação indica que o levantamento realizado pode ser considerado representativo da área de estudo.

Sob uma perspectiva ecológica, a avifauna florestal da ESEC Rio Acre é composta principalmente por duas comunidades distintas: uma associada à floresta aluvial com bambus e/ou palmeiras nas adjacências dos principais cursos d'água que cortam a unidade (Figura 3), e outra associada à floresta aberta com bambus e/ou palmeiras em localidades de altitudes maiores, solos com melhor drenagem e relevos mais acidentados (Figura 4). A principal diferença entre esses dois tipos de fitofisionomia é a densidade das manchas de bambus do gênero Guadua, que é muito mais elevada em terrenos aluviais do que em terrenos com boa drenagem, onde outras espécies arbóreas, incluindo vários tipos de palmeiras, passam a competir com o bambu pela dominância

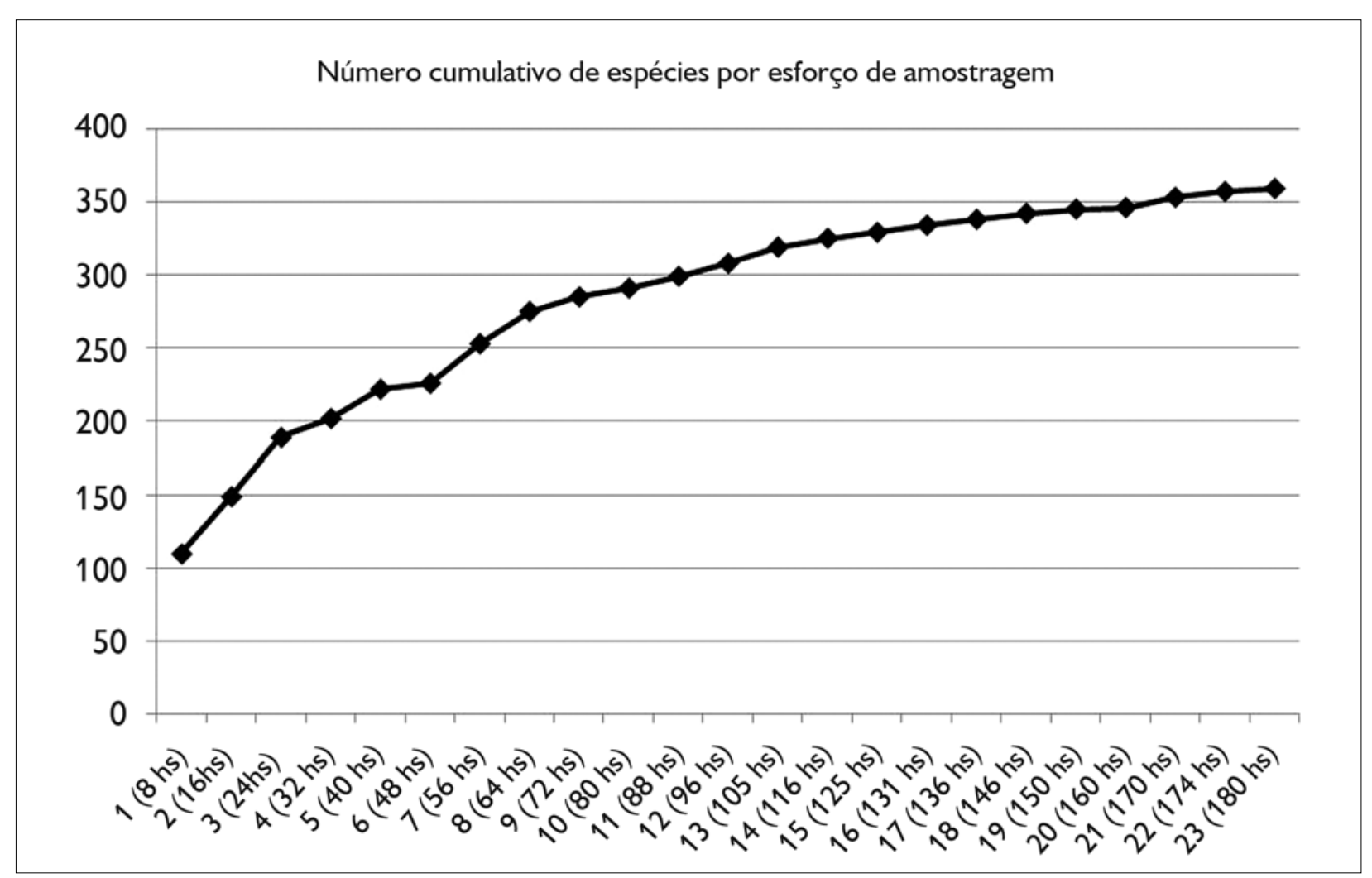

Figura 2. Número de espécies de aves (eixo Y), por dia e número de horas cumulativos de amostragem (eixo X), obtido por um levantamento qualitativo de 180 horas de observação na ESEC Rio Acre, município de Assis Brasil (estado do Acre), entre 13 e 24 de agosto de 2005 $(0-96$ h) e 3 a 16 de fevereiro de 2006 ( $97-180$ h).

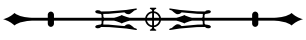




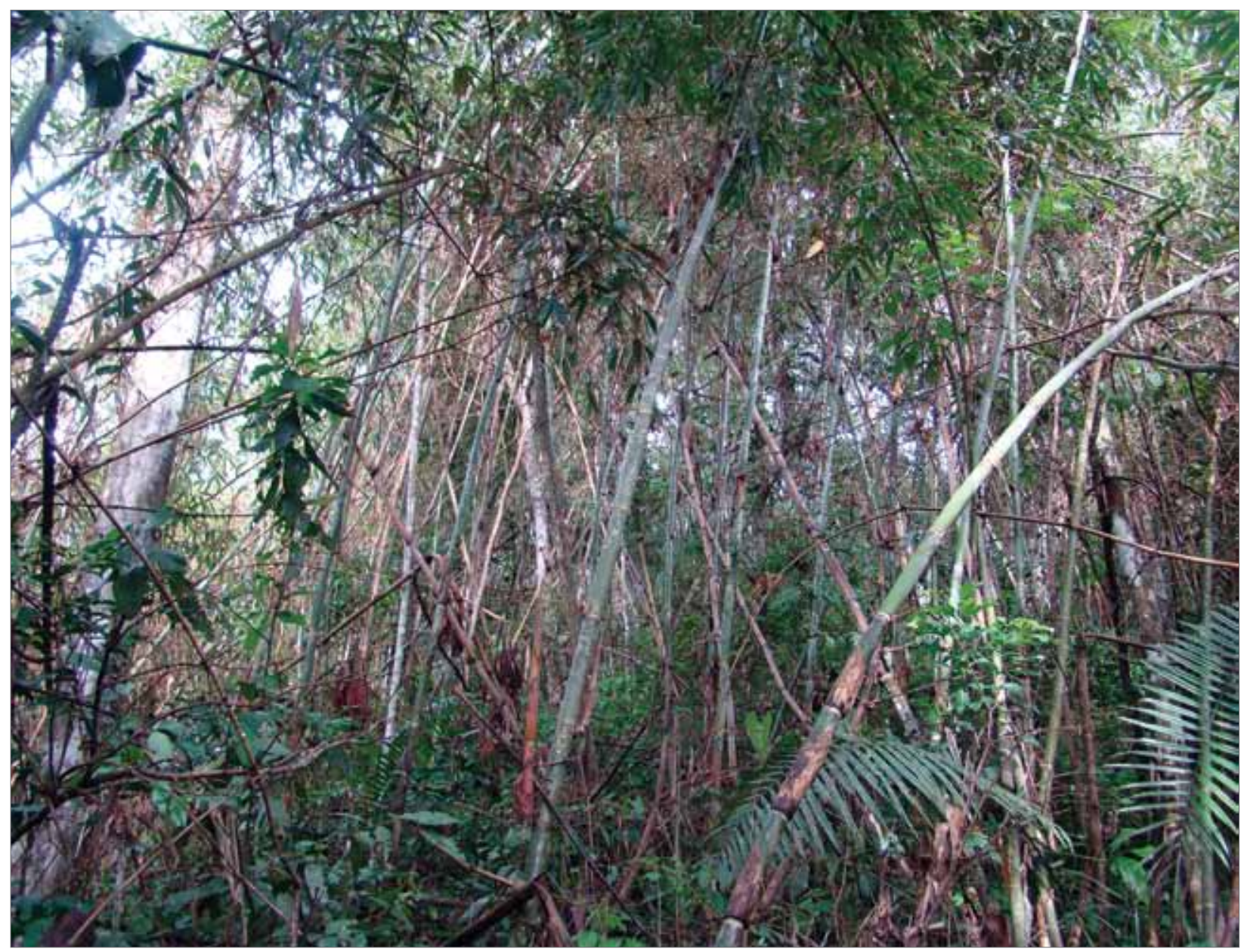

Figura 3. Floresta aluvial com bambus e/ou palmeiras nas adjacências dos principais cursos d'água. Foto: E. Guilherme.

da comunidade. Além destas duas principais fitofisionomias florestais, um terceiro tipo de ambiente foi utilizado por um número menor de espécies da avifauna da ESEC Rio Acre: praias e margens dos principais cursos d'água que cortam a ESEC Rio Acre, denominado daqui em diante como ambiente ripário (Figura 5).

A maior parte das espécies registradas na ESEC Rio Acre (71\%) ocorre em floresta aluvial com predominância de bambus e/ou palmeiras, enquanto cerca de 52\% ocorrem em floresta aberta com bambus e/ou palmeiras em solos drenados (Tabela 1). Um contingente também significativo de 110 espécies (30\% do total) ocorre indistintamente nestes dois tipos de fitofisionomias, não mostrando preferências claras entre elas. Finalmente, apenas 7\% da avifauna da ESEC Rio Acre pode ser considerada não florestal, estando na sua totalidade associada ao ambiente ripário (Tabela 1).

\section{REGISTROS DE RELEVÂNCIA BIOGEOGRÁFICA E PARA A CONSERVAÇÃO}

Do total de 365 espécies registradas na ESEC Rio Acre e vizinhanças imediatas, 71 podem ser consideradas de especial interesse para a conservação em função de sua presença em listas de espécies ameaçadas, distribuições restritas, endemismo, hábitos migratórios, raridade e presença anteriormente não documentada em território

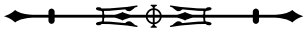




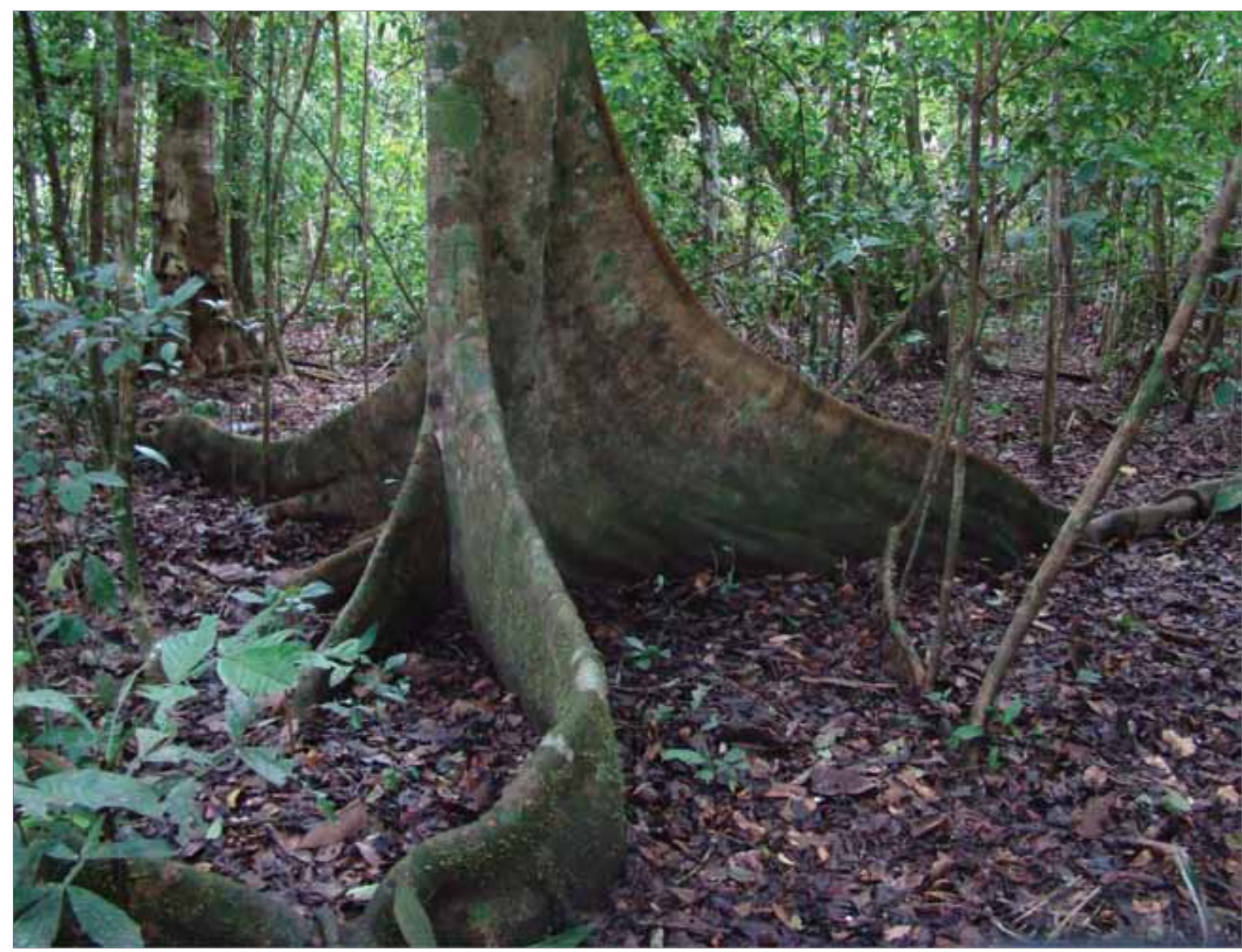

Figura 4. Floresta aberta com bambus e/ou palmeiras em localidades de altitudes maiores, solos com melhor drenagem e relevos mais acidentados. Foto: E. Guilherme.

brasileiro (Aleixo \& Guilherme, 2008; Apêndice). Abaixo, os registros mais relevantes do ponto de vista biogeográfico para 41 destas espécies são discutidos em detalhes.

\section{Família Tinamidae}

Crypturellus obsoletus (Temminck, 1815) - Espécie de distribuição disjunta na América do Sul (Hoyo et al., 1992), é representada por populações isoladas na calha sul da Amazônia brasileira, principalmente a leste do rio Madeira (Sick, 1997). Ocorre também no sudeste do Peru (Schulenberg et al., 2007) e nas regiões central e norte da Bolívia (Remsen \& Traylor, 1989). No Acre, havia sido registrada previamente somente no alto Juruá (Whittaker et al., 2002). O registro da vocalização típica de C. obsoletus na ESEC representa o primeiro para o leste do Acre e o segundo para a Amazônia sul-ocidental brasileira. Embora espécimes não tenham sido coletados, é muito provável que os registros obtidos no Acre, devido à sua distribuição altamente disjunta, refiram-se a uma subespécie ou subespécies distintas daquela encontrada no restante da Amazônia brasileira, C. o. griseiventris (Salvadori, 1895) (Hoyo et al., 1992).

Crypturellus atrocapillus (Tschudi, 1844) - Conhecida originalmente apenas no sudeste do Peru (Terborgh et al., 1984; Schulenberg et al., 2007) e região centro-norte da Bolívia (Remsen \& Traylor, 1989). No Brasil, esta espécie

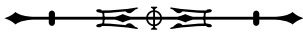




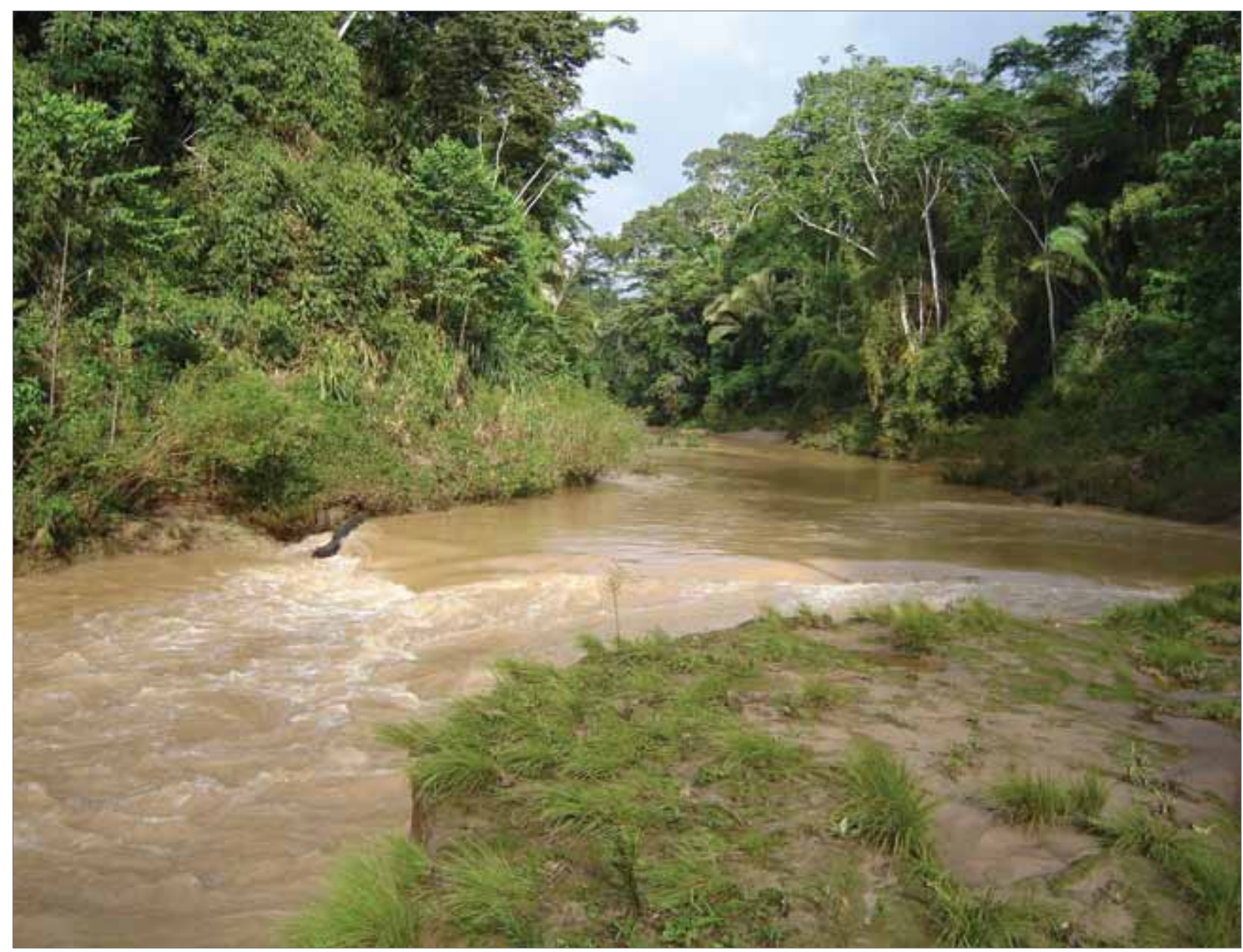

Figura 5. Ambiente ripário. Foto: E. Guilherme.

foi registrada pela primeira vez no município de Marechal Taumaturgo, no alto rio Juruá (Sick, 1997; Whittaker \& Oren, 1999). A observação de C. atrocapillus na ESEC Rio Acre representou o segundo registro confirmado desta espécie para o território brasileiro. Após este registro, diversos outros foram realizados pelo segundo autor (EG) em várias localidades do estado do Acre, incluindo o Parque Zoobotânico da Universidade Federal do Acre, as florestas com bambus no leste do estado e a foz do rio Chandless, no alto Purus (Guilherme \& Dantas, 2008; Guilherme \& Santos, 2009). Estes registros em conjunto indicam que $C$. atrocapillus é bastante comum na região e ocorre em todo o estado do Acre.
Família Accipitridae

Percnohierax leucorrhous (Quoy \& Gaimard, 1824) - No dia 15 de agosto de 2005, um indivíduo adulto exibindo as características típicas desta espécie (Schulenberg et al., 2007) foi avistado pelo primeiro autor (AA) ao longo de vários minutos pairando sobre a floresta aluvial nas proximidades do rio Acre. Este registro é o primeiro desta espécie, associada a regiões montanhosas nos Andes e na Mata Atlântica, para o Acre e a Amazônia brasileira, embora ela já tenha sido registrada nas terras baixas vizinhas ao sopé dos Andes no Peru (Schulenberg et al., 2007). Possivelmente, o indivíduo observado tratava-se de um vagante que chegou à ESEC Rio Acre aproveitando a

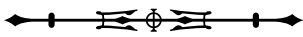


Tabela 1. Número total de espécies, exclusivas e de especial interesse para conservação registradas em cada tipo de fitofisionomia reconhecida como relevante para a avifauna da ESEC Rio Acre.

\begin{tabular}{l|c|c|c}
\hline \multicolumn{1}{c|}{ Tipo de fitofisionomia } & Número de espécies total ${ }^{1}$ & Número de espécies exclusivas ${ }^{2}$ & $\begin{array}{c}\text { Número de espécies de especial } \\
\text { interesse para conservação }^{3}\end{array}$ \\
\hline $\begin{array}{l}\text { Floresta aluvial de bambus } \\
\text { e/ou palmeiras }\end{array}$ & $259(70,9 \%)$ & $149(57,5 \%)$ & $50(70,4 \%)$ \\
\hline $\begin{array}{l}\text { Floresta aberta de bambus } \\
\text { e/ou palmeiras }\end{array}$ & $189(51,7 \%)$ & $79(42,8 \%)$ & $35(49,2 \%)$ \\
\hline $\begin{array}{l}\text { Ambiente ripário - praias e } \\
\text { margens dos rios }\end{array}$ & $27(7,3 \%)$ & $27(100 \%)$ & $4(5,6 \%)$ \\
\hline
\end{tabular}

1 Número total de espécies registrado no respectivo tipo de vegetação. Valores entre parênteses denotam porcentagem em relação ao número total de espécies registrado em todas as vegetações e localidades da ESEC Rio Acre $(n=365)$.

2 Número de espécies exclusivas do respectivo tipo de vegetação. Valores entre parênteses denotam porcentagem em relação ao número total de espécies registrado na respectiva fitofisionomia.

3 Número total de espécies de especial interesse para a conservação registrado no respectivo tipo de vegetação (ver Apêndice para definição das espécies de especial interesse para a conservação). Valores entre parênteses denotam porcentagem em relação ao número total de espécies de especial interesse para a conservação registrado em todas as vegetações e localidades da ESEC Rio Acre $(n=71)$.

entrada de uma forte frente fria, que havia chegado à área há apenas dois dias.

\section{Família Psittacidae}

Primolius couloni (Sclater, 1876) - Esta espécie foi registrada pela primeira vez no estado do Acre por Parker \& Remsen (1987), através da observação de alguns indivíduos às proximidades da cidade de Capixaba, no leste do Acre. Posteriormente, foi vista na região de Plácido de Castro (Forrester, 1993), e no município de Rio Branco (Tobias \& Brightsmith, 2007; Guilherme \& Santos, 2009), também no leste do estado. Na região do alto Juruá, esta espécie parece ser relativamente comum em floresta aluvial (Whittaker \& Oren, 1999). Em 2004, EG coletou na região central do estado (Seringal Sardinha) a segunda pele de P. couloni para o estado do Acre (Tobias \& Brightsmith, 2007; Guilherme \& Santos, 2009). 0 espécime coletado estava associado a um grupo de seis indivíduos que se alimentavam de frutos de mandioca (Manihot esculenta) em um pequeno roçado localizado à beira da BR-364, entre as cidades de Manuel Urbano e Feijó. Na ESEC Rio Acre, P. couloni é uma espécie frequente tanto em floresta aluvial quanto aberta em terrenos mais altos, tendo sido observada em cinco dos 13 sítios amostrados na unidade (Apêndice). A forma mais frequente de contato com a espécie foi o avistamento de bandos de quatro a oito indivíduos se deslocando em voo principalmente durante o amanhecer e o crepúsculo.

Pyrrhura rupicola (Tschudi, 1844) - Espécie restrita à área de endemismo Inambari (Cracraft, 1985) e cuja distribuição geográfica conhecida se restringia quase que exclusivamente ao sudeste do Peru (Parker, 1982; Schulenberg et al., 2007) e norte da Bolívia (Remsen \& Traylor, 1989; Hoyo et al., 1997). Em território brasileiro, esta espécie só foi registrada no estado do Acre. Os registros incluem uma pele coletada por J. Hidasi nos arredores da cidade de Capixaba (Sick, 1997) e observações realizadas por Forrester (1993) nos arredores de Plácido de Castro. P. rupicola foi observada também na Reserva Extrativista do Alto Juruá (RESEX Alto Juruá), extremo oeste do estado (Whittaker \& Oren, 1999; Whittaker et al., 2002). Na ESEC Rio Acre, P. rupicola é abundante, principalmente em florestas ao longo dos principais cursos d'água. No dia 15 de agosto de 2005, um macho adulto foi coletado por AA em floresta aluvial localizada na margem esquerda do rio Acre (MPEG

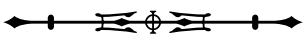


58825). Este espécime é o segundo deste táxon coletado em território brasileiro.

Nannopsittaca dachilleae O’Neill, Munn \& Franke, 1991 - Espécie conhecida apenas das terras baixas do sudeste do Peru, noroeste da Bolívia (Clements \& Shany, 2001; Schulenberg et al., 2007), e extremo sudoeste da Amazônia brasileira (estado do Acre) (Whitney \& Oren, 2001a). Os seus primeiros registros no Brasil foram feitos no Parque Nacional da Serra do Divisor, no alto Juruá (Whitney \& Oren, 2001a). O registro de N. dachilleae na ESEC Rio Acre é o segundo para o território brasileiro e representa uma significativa ampliação de sua distribuição em direção ao sudeste das terras baixas da Amazônia Legal.

\section{Família Caprimulgidae}

Caprimulgus sericocaudatus (Cassin, 1849) - Espécie rara, pouco conhecida e de ocorrência pontual na Amazônia (Davis et al., 1991; Aleixo et al., 2000; Schulenberg et al., 2007) e no sudeste do Brasil (Sick, 1997). A gravação da vocalização desta espécie na ESEC Rio Acre representa o primeiro registro para o estado do Acre e estende a sua distribuição para a Amazônia ocidental brasileira.

\section{Família Apodidae}

Chaetura spinicaudus (Temminck, 1839) - O registro mais próximo desta espécie ao sul do rio Amazonas havia sido feito por Gyldenstolpe (1951), no baixo rio Purus, no estado do Amazonas. Recentemente, Rasmussen et al. (2005) registraram este táxon em um fragmento florestal a $30 \mathrm{~km}$ de Rio Branco, estado do Acre. A presença de C. spinicaudus na ESEC representa o registro mais ao sul conhecido, na Amazônia ocidental brasileira.

Chaetura meridionalis Hellmayr, 1907 - Espécie migrante e de população disjunta na América do Sul (Sick, 1997; Hoyo et al., 1999). A observação desta espécie na ESEC Rio Acre representa o primeiro registro para o estado do Acre. Muito provavelmente, os indivíduos observados na ESEC eram migrantes austrais.
Família Trochilidae

Amazilia lactea bartletti (Sclater \& Salvin, 1866) - Esta subespécie é de ocorrência restrita às terras baixas da Amazônia sul-ocidental, incluindo o sudeste do Peru, o norte da Bolívia e o sudoeste da Amazônia Legal (Hoyo et al., 1999). Até o momento, todos os registros deste táxon em território brasileiro foram feitos no estado do Acre (Pinto \& Camargo, 1954; Guilherme, 2001; Whittaker et al., 2002; Guilherme, 2004; Rasmussen et al., 2005). Na ESEC Rio Acre, este táxon foi comumente visto em florestas aluviais, visitando flores de marantáceas localizadas na margem esquerda do rio Acre.

\section{Família Galbulidae}

Galbalcyrhynchus purusianus Goeldi, 1904 - Espécie restrita à área de endemismo Inambari (Cracraft, 1985). No estado do Acre, Galbalcyrhynchus purusianus já foi registrado no alto Juruá e no alto Purus (Whittaker et al., 2002; Guilherme \& Dantas, 2008). Na ESEC Rio Acre, esta espécie é comum em floresta aluvial ao longo do rio Acre. $O$ registro de $G$. purusianus na ESEC é o primeiro para o extremo leste do estado do Acre.

\section{Família Bucconidae}

Malacoptila semicincta Todd, 1925 - Restrita à área de endemismo Inambari (Haffer, 1978, 1987; Cracraft, 1985). Ocorre no sudeste do Peru (Servat, 1996; Schulenberg et al., 2007) e norte da Bolívia (Gyldenstolpe, 1945; Remsen \& Traylor, 1989). No Acre, era conhecida apenas do alto Juruá (Novaes, 1957; Whittaker et al., 2002). Na ESEC Rio Acre, foram coletados dois espécimes (MPEG 58846 e 59792) em sub-bosque de floresta ombrófila com palmeiras. Os registros de M. semicincta na ESEC foram os primeiros para o leste do Acre. Posteriormente, esta espécie foi registrada em diversas localidades do estado, incluindo a região do alto Purus e do rio Liberdade, no oeste do Acre (Guilherme, 2007; Guilherme \& Dantas, 2008). Estes novos registros, em conjunto, indicam que esta espécie é comum em todo o estado do Acre.

Nonnula sclateri Hellmayr, 1907 - Espécie pouco conhecida, ocorre no sudeste do Peru (Davis et al., 
1991; Schulenberg et al., 2007) e no Departamento de Pando, na Bolívia (Parker \& Remsen, 1987). No Acre, esta espécie era previamente conhecida apenas da bacia do rio Juruá (Novaes, 1957; Whittaker \& Oren, 1999; Whittaker et al., 2002). Na ESEC Rio Acre, N. sclateri foi documentada através do registro da vocalização de um indivíduo pousado num bambuzal bastante denso. Este registro foi o primeiro desta espécie para o leste do Acre. Posteriormente, EG coletou vários indivíduos de N. sclateri em diversas localidades do estado do Acre (Guilherme, 2007; Guilherme \& Dantas, 2008).

Monasa flavirostris Strickland, 1850 - Espécie pouco conhecida, associada às florestas dominadas por bambus (Stotz et al., 1996). No Acre, só havia sido registrada no alto Juruá (Whittaker et al., 2002) e na Fazenda Experimental Catuaba, próxima de Rio Branco (Rasmussen et al., 2005). Recentemente, Guilherme \& Santos (2009) registraram esta espécie em uma floresta dominada por bambus no município de Rio Branco, no leste do estado. O registro de $M$. flavirostris na ESEC Rio Acre indica que a espécie, apesar de rara, ocorre em todo o estado do Acre.

\section{Família Capitonidae}

Eubucco tucinkae (Seilern, 1913) - Espécie de ocorrência restrita à área de endemismo Inambari (Cracraft, 1985), foi considerada, por algum tempo, como sendo endêmica do território peruano até ser registrada em território boliviano (Parker et al., 1991). No Brasil, esta espécie só é conhecida a partir dos registros feitos no estado do Acre (Whittaker \& Oren, 1999; Whitney \& Oren, 2001b; Guilherme, 2007). $\mathrm{Na}$ ESEC Rio Acre, E. tucinkae foi registrada uma única vez em floresta aluvial de estágio sucessional inicial, na margem esquerda do rio Acre. Esse registro é o primeiro desta espécie para a região leste do Acre e amplia a sua ocorrência em território brasileiro.

\section{Família Ramphastidae}

Aulacorhynchus atrogularis (Sturm \& Sturm, 1841) - Espécie de distribuição centrada nas florestas andinas e pré-andinas da Bolívia, Peru e Equador (Sick, 1997; Schulenberg et al., 2007), foi registrada pela primeira vez no Brasil apenas em 1989, nas proximidades de Plácido de Castro, estado do Acre (Forrester, 1993). Outras observações desta espécie foram feitas no alto rio Juruá (Whittaker \& Oren, 1999), no oeste do Acre, e no alto Purus (Guilherme \& Dantas, 2008), na região central do estado. Nosso registro para a ESEC Rio Acre representa a quarta localidade de ocorrência desta espécie em território brasileiro.

\section{Família Picidae}

Celeus spectabilis Sclater \& Salvin, 1880 - Restrita às florestas dominadas por bambus na Amazônia ocidental (Stotz et al., 1996). No Brasil, só havia sido registrada no alto Juruá (Whittaker \& Oren, 1999). Em 2004, AA coletou, no baixo rio Paratari (Parque Nacional da Serra do Divisor), o primeiro espécime para o Brasil (MPEG 58371). O registro de $C$. spectabilis na ESEC Rio Acre foi o primeiro desta espécie para o leste do Acre (bacia do rio Purus). Posteriormente, outros espécimes de C. spectabilis foram coletados em uma floresta dominada por bambus próxima a Rio Branco (Guilherme \& Santos, 2009). O conjunto destas observações demonstra que esta espécie é comum nas florestas com bambus em todo o estado do Acre.

\section{Família Thamnophilidae}

Cymbilaimus sanctaemariae Gyldenstolpe, 1941 - Espécie associada às florestas dominadas por bambus e de ocorrência restrita à calha sul do Amazonas (Stotz et al., 1996; Parker et al., 1997; Hoyo et al., 2003). O primeiro registro deste táxon para o Acre foi feito a partir de alguns exemplares coletados por J. Hidasi no Seringal Nova Empresa, próximo de Rio Branco (Pierpont \& Fitzpatrick, 1983; LSUMZ 68109; Parker et al., 1997; MZUSP 66060 e 66061). Somente na década de 1990 este táxon foi novamente registrado no estado, através de gravações feitas em manchas de bambus no alto Juruá (Whittaker \& Oren, 1999; Whittaker et al., 2002). Na ESEC Rio Acre, dois espécimes foram coletados em floresta aluvial com a presença de bambus (MPEG 58888 e 58889). Os 
registros de C. sanctaemariae na ESEC Rio Acre, associados a outros realizados posteriormente em diversas regiões do estado (Guilherme \& Dantas, 2008; Guilherme \& Santos, 2009), indicam que esta espécie é bastante comum nas florestas ombrófilas dominadas por bambus em todo o Acre.

Herpsilochmus rufimarginatus (Temminck, 1822) Os registros desta espécie mais próximos do Acre foram feitos no sudeste do Peru e nas regiões central e norte da Bolívia (Remsen \& Traylor, 1989; Bates et al., 1989; Clements \& Shany, 2001; Schulenberg et al., 2007). Um espécime foi gravado e coletado no dossel de floresta ombrófila aberta na ESEC Rio Acre (MPEG 59862). Este registro representa o primeiro para o Acre e o mais ocidental dentro da Amazônia brasileira.

Cercomacra manu Fitzpatrick \& Willard, 1990 Espécie associada a florestas com bambus (Stotz et al., 1996) e de distribuição local na Amazônia brasileira (Ridgely \& Tudor, 1994). No Acre, só havia sido registrada no alto Juruá (Whittaker \& Oren, 1999; Whittaker et al., 2002). Em 2004, AA coletou um espécime (MPEG 58384) no Parque Nacional da Serra do Divisor, margem oeste do rio Juruá (município de Marechal Taumaturgo). A gravação da vocalização de C. manu na ESEC Rio Acre representou o primeiro desta espécie para o leste do Acre. Após este registro, dois espécimes foram coletados (MPEG 61336-37) em uma floresta com bambus no ramal Jarinal, no município de Rio Branco (Guilherme \& Santos, 2009).

Hypocnemis subflava collinsi Cherrie, 1916 - Nova combinação taxonômica proposta recentemente por Isler et al. (2007). Anteriormente os epítetos subflava e collinsi designavam subespécies de $H$. cantator (Ridgely \& Tudor, 1994). Entretanto, baseados em diferenças morfológicas e vocais, Isler et al. (2007) elevaram subflava à categoria de espécie plena, incluindo neste grupo as subespécies H. s. subflava Cabanis, 1873 e H. s. collinsi. Esta última, de ocorrência restrita ao sudeste do Peru e noroeste da Bolívia (Ridgely \& Tudor, 1994; Schulenberg et al., 2007), foi registrada em 1997 na Fazenda Experimental Catuaba (leste do Acre) por B. M. Whitney (Isler et al., 2007), tendo sido esta a primeira observação da espécie $H$. subflava no Brasil. Na ESEC Rio Acre, dois espécimes de Hypocnemis subflava collinsi foram coletados e vários gravados no sub-bosque de floresta ombrófila aberta com palmeiras (MPEG 59855 e 58903). Os espécimes oriundos da ESEC são os primeiros coletados em território brasileiro. Recentemente, novos registros de H. s. collinsi foram feitos em uma floresta com bambus a sudoeste da cidade de Rio Branco (Guilherme \& Santos, 2009).

Percnostola lophotes Hellmayr \& Seilern, 1914 - Esta ave é associada às florestas com bambus no sudeste do Peru (Parker, 1982; Terborgh et al., 1984; Davis et al., 1991; Servat, 1996; Kratter, 1997) e norte da Bolívia (Parker \& Remsen, 1987; Parker et al., 1991). O primeiro registro desta espécie em território brasileiro foi feito no alto Juruá, estado do Acre (Whittaker \& Oren, 1999; Whittaker et al., 2002). Na ESEC Rio Acre, Percnostola lophotes foi comumente observada, sempre associada a manchas extensas de bambu, principalmente em floresta aluvial.

Myrmeciza goeldii (Snethlage, 1908) - Espécie restrita à área de endemismo Inambari (Cracraft, 1985) e associada às florestas ombrófilas com bambus (Stotz et al., 1996; Kratter, 1997) e florestas ombrófilas aluviais (várzeas). Ocorre no sudeste do Peru (Parker, 1982; Terborgh et al., 1984; Schulenberg et al., 2007) e no norte da Bolívia (Parker \& Remsen, 1987; Parker et al., 1991). Conhecida no Brasil até bem pouco tempo apenas a partir do holótipo descrito por E. Snethlage, proveniente da localidade 'Bom Lugar', no médio Purus (Gyldenstolpe, 1951). No Acre, esta espécie foi registrada pela primeira vez no alto Juruá (Whittaker \& Oren, 1999). Em 9 de junho de 2000, EG capturou e anilhou (anilha H41511) uma fêmea no Parque Zoobotânico da Universidade Federal do Acre (Guilherme, 2001). Na ESEC Rio Acre, foram coletados quatro espécimes (MPEG 58906, 58907, 59844 e 59845) em floresta aluvial. Trata-se de uma espécie de vocalização facilmente reconhecível e de ocorrência em todo o estado do Acre (Whittaker \& Oren, 1999; Guilherme, 2001; Guilherme \& Dantas, 2008; Guilherme \& Santos, 2009). 
Família Formicariidae

Formicarius rufifrons Blake, 1957 - Esta espécie era conhecida, até bem pouco tempo, apenas do sudeste do Peru, Departamento de Madre de Dios (Ridgely \& Tudor, 1994; Schulenberg et al., 2007). Só foi registrada no Brasil em 1995 na região do alto Juruá (Whittaker \& Oren, 1999; Whittaker et al. , 2002). No dia 17 de agosto de 2005, um indivíduo foi ouvido vocalizando o canto territorial típico da espécie próximo à base de apoio do IBAMA na ESEC. O registro deste táxon para a ESEC Rio Acre é o segundo para o território brasileiro e o primeiro para a bacia do rio Purus (leste do estado do Acre).

\section{Família Scleruridae}

Sclerurus albigularis Sclater \& Salvin, 1869 - Foi registrada no Brasil pela primeira vez no estado de Rondônia, em Cachoeira de Nazaré (Stotz et al., 1997). Posteriormente, foi observada no alto Juruá, oeste do Acre (Whittaker \& Oren, 1999; Whittaker et al., 2002). Coletamos os primeiros dois indivíduos desta espécie para o estado do Acre (MPEG 58881 e 58882), além de gravar vários indivíduos. Os espécimes oriundos da ESEC Rio Acre representam o primeiro registro deste táxon para a bacia do rio Purus.

\section{Família Dendrocolaptidae}

Glyphorynchus spirurus albigularis Chapman, 1923 - Esta subespécie é claramente distinta das demais agrupadas dentro de G. spirurus e distinguida principalmente pelo mento e garganta brancos (Gyldenstolpe, 1945). Ocorre no Peru (Clements \& Shany, 2001 [Prancha 67]; Schulenberg et al., 2007) e Bolívia (Gyldenstolpe, 1945). Coletamos dois exemplares deste táxon (MPEG 58874 e 59810) em sub-bosque de floresta ombrófila com palmeiras dentro dos limites da ESEC Rio Acre. Os espécimes oriundos da ESEC constituem os primeiros registros deste táxon em território brasileiro.

Xiphorhynchus chunchotambo (Tschudi, 1844) Espécie conhecida do sopé dos Andes do sul da Colômbia, sudeste do Peru ao centro/norte da Bolívia (Hoyo et al., 2003). Em agosto de 2005, AA gravou a vocalização e coletou dois espécimes (MPEG 58864 e 58865) em sub-bosque de floresta aluvial com bambus na ESEC Rio Acre. Em fevereiro de 2006, foi coletado mais um espécime em um novo ponto de amostragem dentro da unidade (MPEG 59806). Os registros de Xiphorhynchus chunchotambo na ESEC Rio Acre, em conjunto com outros feitos por EG em diversas localidades do estado do Acre, representaram os primeiros deste táxon para o território brasileiro (Guilherme \& Aleixo, 2008).

\section{Família Furnariidae}

Synallaxis cherriei Gyldenstolpe, 1930 - Espécie de ocorrência comum na Amazônia sul-oriental do Brasil, no norte de Mato Grosso, região de Alta Floresta (Zimmer et al., 1997) e no interflúvio Xingu/Araguaia, no Pará (Oren \& Silva, 1987; Aleixo et al., 2000). Na Amazônia ocidental, Synallaxis cherriei só foi registrada pontualmente no sudeste do Peru (Schulenberg et al., 2007) e norte da Bolívia (Tobias \& Seddon, 2007). No Acre, esta espécie só havia sido registrada na Reserva Extrativista do Alto Juruá (Whittaker et al., 2002). 0 registro de Synallaxis cherriei na ESEC Rio Acre foi o primeiro para o leste do Acre e o segundo para Amazônia sul-ocidental brasileira. Posteriormente, esta espécie foi registrada em uma floresta com bambus, no município de Rio Branco, leste do Acre (Guilherme \& Santos, 2009).

Simoxenops ucayalae (Chapman, 1928) - Espécie associada às florestas ombrófilas dominadas por bambus no sudeste do Peru (Parker, 1982; Terborgh et al., 1984; Servat, 1996; Kratter, 1997) e norte da Bolívia (Parker \& Remsen, 1987). Na Amazônia brasileira, S. ucayalae tem sido registrada pontualmente no Acre, Pará e norte do Mato Grosso (Aleixo et al., 2000; Zimmer et al., 1997). Apesar de Novaes (1978) ter associado um espécime, sem procedência comprovada, às florestas do Acre, a comprovação da presença de S. ucayalae no estado só foi confirmada a partir de cinco exemplares coletados pela equipe do MPEG no rio Tejo, alto Juruá (Whittaker 
\& Oren, 1999; Whittaker et al., 2002), no oeste do Acre. O espécime coletado em floresta ombrófila com bambus na ESEC Rio Acre (MPEG 58862) foi o primeiro deste táxon para a região leste do estado do Acre. Após este registro, Guilherme \& Santos (2009) coletaram mais um espécime deste táxon no município de Rio Branco, também no leste do Acre.

Philydor rufum bolivianum Berlepsch, 1907 - Este táxon ocorre desde o sopé dos Andes, no Equador até a região central da Bolívia (Hoyo et al., 2003). Em 14 de agosto de 2005, um indivíduo foi capturado numa rede de neblina, em floresta aluvial, e coletado (MPEG 58861). Este espécime representa o primeiro registro deste táxon bastante diferenciado em território brasileiro (Hoyo et al., 2003).

\section{Família Tyrannidae}

Lophotriccus eulophotes Todd, 1925 - Espécie restrita à área de endemismo Inambari (Cracraft, 1985) e associada às florestas dominadas por bambus (Stotz et al., 1996). No Acre, foi registrada pela primeira vez no alto Juruá (Whittaker \& Oren, 1999). Em 2004, AA coletou dois espécimes no Parque Nacional da Serra do Divisor (MPEG 58021 e 58387). Este táxon foi registrado também na Fazenda Experimental Catuaba, no leste do estado (Rasmussen et al., 2005). Na ESEC Rio Acre, foram gravados e coletados dois espécimes (MPEG 59876 e 59877) em sub-bosque de floresta ombrófila aberta com bambus. Posteriormente, EG coletou outros espécimes de Lophotriccus eulophotes nos municípios de Porto Acre, Reserva Humaitá (MPEG 60030 e 60031) e Bujari, na Floresta Estadual do Antimary (MPEG 60758). Estes registros demonstram que $L$. eulophotes é comum nas florestas com bambus em todo o estado do Acre.

Hemitriccus flammulatus Berlepsch, 1901 - Esta espécie é associada às florestas dominadas por bambus na Amazônia ocidental (Stotz et al., 1996). Ocorre a partir do sopé dos Andes, no Peru e na Bolívia, em direção às terras baixas da Amazônia (Remsen \& Traylor, 1989; Ridgely \& Tudor, 1994; Schulenberg et al., 2007). No
Acre, já tinha sido registrada nos arredores de Rio Branco (Guilherme, 2001; Rasmussen et al., 2005) e também no alto Juruá (Whittaker et al., 2002). Na ESEC Rio Acre, foram coletados três indivíduos deste táxon (MPEG 58919, 59874 e 59875) em sub-bosque de floresta com bambus. Trata-se de uma espécie bastante comum nas florestas associadas com bambus em todo o estado do Acre (Guilherme, 2001, 2007; Guilherme \& Dantas, 2008; Guilherme \& Santos, 2009).

Cnipodectes superrufus Lane, Servat, Valqui \& Lambert, 2007 - Esta espécie foi recentemente descrita com base em registros feitos em florestas ombrófilas dominadas por bambus, localizadas no sudeste do Peru (Lane et al., 2007). Mesmo antes de sua descrição formal, vários indivíduos de Cnipodectes superrufus já haviam sido coletados e anilhados por EG no Parque Zoobotânico da Universidade Federal do Acre desde 1998, tendo sido identificado, à época, como Cnipodectes subbrunneus (Guilherme, 2001; Tobias et al., 2008). Em 15 de agosto de 2005, AA observou e gravou esta espécie em manchas de bambus próximas à base do IBAMA, na ESEC Rio Acre. Este registro foi o segundo para esta espécie em território brasileiro (Tobias et al., 2008). Posteriormente, a espécie foi registrada em uma floresta com bambus a aproximadamente $70 \mathrm{~km}$ a oeste da cidade de Rio Branco (ramal Jarinal), no leste do Acre (Tobias et al., 2008; Guilherme \& Santos, 2009).

Ramphotrigon megacephalum (Swainson, 1835) Espécie de ocorrência aparentemente disjunta na América do Sul (Ridgely \& Tudor, 1994), mas comum na Amazônia sul ocidental (Remsen \& Traylor, 1989; Schulenberg et al., 2007). Apesar disso, Ramphotrigon megacephalum foi registrada poucas vezes na Amazônia brasileira (Parker et al., 1997; Pacheco, 1995). No Acre, só havia sido observada na Reserva Extrativista do Alto Juruá (Whittaker et al., 2002). AA coletou em 2004 um espécime no Parque Nacional da Serra do Divisor (MPEG 58023), próximo de Cruzeiro do Sul. O registro de $R$. megacephalum na ESEC Rio Acre foi o primeiro para o leste do Acre. 


\section{Família Cotingidae}

Conioptilon mcilhennyi Lowery \& O'Neill, 1966 - Embora este táxon tenha sido descrito em uma localidade peruana próxima à fronteira com o Brasil (Lowery \& O'Neill, 1966), ele só foi registrado em território brasileiro em 1994 no alto Juruá (Whittaker \& Oren, 1999; Whittaker et al., 2002). Na ESEC Rio Acre, esta espécie é bastante comum em floresta aluvial, de onde provém o primeiro espécime coletado no leste do estado (MPEG 58938). Posteriormente, EG obteve outros registros de Conioptilon mcilhennyi nos municípios de Bujari e Porto Acre. Os registros aqui relatados ampliam significativamente a distribuição geográfica deste táxon em direção às terras baixas da Amazônia brasileira.

\section{Família Pipridae}

Neopelma sulphureiventer (Hellmayr, 1903) - Espécie com distribuição geográfica restrita à área de endemismo Inambari (Haffer, 1978; Cracraft, 1985) e associada às florestas dominadas por bambus na calha sul do Solimões (Stotz et al., 1996). Ocorre no sudeste do Peru (Terborgh et al., 1984; Servat, 1996; Schulenberg et al., 2007) e nas regiões central e norte da Bolívia (Remsen \& Traylor, 1989). No Acre, já foi registrada em praticamente todo o estado (Whittaker \& Oren, 1999; Whittaker et al., 2002; Guilherme, 2007; Guilherme \& Dantas, 2008; Guilherme \& Santos, 2009). Na ESEC Rio Acre, foram coletados três espécimes (MPEG 58927, 58928 e 58929) no subbosque de floresta ombrófila com bambus.

\section{Família Tityridae}

Pachyramphus xanthogenys Salvadori \& Festa, 1898 - Espécie de distribuição local no extremo oeste da Amazônia, a partir do sopé dos Andes no Peru, Equador e extremo-sul da Colômbia (Ridgely \& Tudor, 1994; Hoyo et al., 2004). Tem sido considerada por alguns autores como subespécie de Pachyramphus viridis (Vieillot, 1816), cuja distribuição abrange quase todo o Brasil (Peters, 1979; Sick, 1997). Entretanto, tendo como base a sua distribuição disjunta, bem como as diferenças de plumagem e de vocalização em relação a $P$. viridis, adotamos, assim como o CBRO (2009), a classificação de Hoyo et al. (2004), que consideram Pachyramphus xanthogenys como espécie válida. Em 25 de agosto de 2005, AA coletou na ESEC um indivíduo macho adulto (MPEG 58925) em sub-bosque de floresta ombrófila aberta com bambus. No ano seguinte, em 8 de setembro de 2006, EG coletou um espécime jovem (não sexado) em uma área de vegetação secundária, a aproximadamente $60 \mathrm{~km}$ a oeste de Rio Branco. Esses registros de $P$. xanthogenys em duas localidades do estado do Acre foram os primeiros para esta espécie em território brasileiro (Aleixo et al., 2008).

\section{Família Vireonidae}

Vireo flavoviridis (Cassin, 1851) - No estado do Acre, a presença de $V$. flavoviridis foi registrada pela primeira vez em 1995, no alto Juruá (foz do igarapé São João e no rio Amônia, localidade Quieto) (Whittaker \& Oren, 1999). Em março de 1997, B. M. Whitney registrou a presença de nove indivíduos desta espécie dentro dos limites do Parque Nacional da Serra do Divisor (Whitney \& Pacheco, 2001). O registro da vocalização de um único indivíduo de V. flavoviridis na ESEC Rio Acre, em fevereiro de 2006, em floresta aluvial, representa o primeiro registro desta espécie para o leste do estado do Acre. Adicionalmente a estes registros, EG coletou em 2007 os dois primeiros indivíduos desta espécie (MPEG 62172 e 63820) dentro dos limites territoriais do Acre, ambos no oeste do estado (Guilherme \& Borges, 2008). Os sítios de nidificação de V. flavoviridis estão concentrados na região compreendida entre o norte do México (incluindo as ilhas adjacentes) e o centro do Panamá (Restall et al., 2006). Segundo Whitney \& Pacheco (2001), a compilação dos registros de V. flavoviridis na América do Sul indica a região sudoeste da Amazônia brasileira e as porções adjacentes do Peru e da Bolívia como área de invernada desta espécie no continente sul-americano. 
Família Polioptilidae

Polioptila plumbea (Gmelin, 1788) - Esta espécie é de ampla distribuição no norte da América do Sul, desde a Amazônia até o Panamá e o México (Ridgely \& Tudor, 1994). No Acre, este táxon só havia sido registrado no alto Juruá (Whittaker et al., 2002). O registro desta espécie na ESEC é o segundo para o estado e representa uma considerável extensão da sua distribuição conhecida para o sudeste da Amazônia ocidental brasileira.

\section{Família Emberizidae}

Tiaris sp. - No dia 7 de fevereiro de 2006, um indivíduo vocalizando o canto característico das espécies deste gênero foi gravado por AA em floresta aluvial com bambu, no início da manhã. Infelizmente, não foram obtidos outros registros, avistamentos ou espécimes deste táxon na ESEC Rio Acre para confirmar sua identificação específica. Tiaris obscurus (d'Orbigny \& Lafresnaye, 1837) é registrado com regularidade no Departamento de Madre de Dios, no Peru, vizinho à ESEC Rio Acre, principalmente durante o inverso austral (Bates, 1997). Portanto, o registro aqui reportado é intrigante na medida em que ocorreu no verão austral e em floresta com a presença de bambu, ambiente este selecionado por outra espécie do mesmo gênero já registrada na periferia setentrional e meridional da Amazônia: T. fuliginosus (Wied, 1830). Como discutido por Bates (1997), juntos, T. obscurus e T. fuliginosus, formam um par parapátrico de espécies de distribuição circum-amazônica e tem uma diagnose recíproca difícil, ocorrendo juntos em algumas localidades da América do Sul. Futuros levantamentos e, principalmente, a obtenção de espécimes de Tiaris na região leste do Acre podem esclarecer melhor a distribuição destas duas formas na Amazônia ocidental.

\section{Família Icteridae}

Psarocolius angustifrons alfredi (Des Murs, 1856) - Este táxon é restrito ao leste dos Andes, do sul do Equador às terras-baixas do sudeste do Peru (Ridgely \& Tudor, 1994;
Schulenberg et al., 2007). Em março de 2004, EG coletou um espécime (AC-0065, depositado na Universidade Federal do Acre - UFAC) nos arredores da cidade de Assis Brasil (Guilherme, 2004). Na ESEC Rio Acre, foram coletados mais dois espécimes (MPEG 59899 e 59900) em floresta aluvial ao longo do rio Acre. Trata-se de um táxon bastante comum na região do alto rio Acre, onde foi visto diariamente em bandos de mais de 20 indivíduos, ou sobrevoando ou alimentando-se de frutos na transição entre o ambiente ripário e a floresta aluvial, com a presença abundante de embaúbas (Cecropia sp.) às margens do rio Acre. Os espécimes coletados na região do alto rio Acre representam os primeiros registros de Psarocolius angustifrons alfredi em território brasileiro.

\section{A RELEVÂNCIA DA ESEC RIO ACRE PARA A CONSERVAÇÃO DA AVIFAUNA}

Os resultados obtidos demonstram que a ESEC Rio Acre sustenta uma avifauna altamente diversificada e associada a um tipo de vegetação relativamente pouco representado em território brasileiro: a floresta dominada por bambus e tipos vegetacionais associados (Silveira, 2005). Do total de 365 espécies de aves registradas na ESEC Rio Acre, 71 (cerca de 19,4\%) foram consideradas de especial interesse (Apêndice). A ESEC Rio Acre tem um papel chave na preservação de populações destas espécies, na sua maior parte com distribuição centrada fora do território brasileiro e associadas a ecossistemas de ocorrência marginal no Brasil, como florestas de bambus e florestas abertas prémontanas Andinas.

Foram identificadas na ESEC Rio Acre apenas três comunidades de aves distintas: 1) uma associada à floresta aluvial de bambus e/ou palmeiras; 2) outra associada à floresta aberta com bambus e/ou palmeiras, em solos com melhor drenagem e relevos mais acidentados e 3) uma menos representativa, em ambiente não florestal ripário (Tabela 1). A floresta aluvial é aquela mais rica em espécies e que também abriga a maior proporção de espécies de especial interesse para conservação, seguida pela floresta 
aberta com bambus e/ou palmeiras e pelo ambiente ripário. Portanto, o tipo fitofisionômico mais rico em espécies de aves de especial interesse para conservação na ESEC Rio Acre é justamente aquele ao lado dos principais cursos d'água, que constituem praticamente a única via de acesso à unidade e em cujas margens, no lado peruano, já existem acampamentos madeireiros estabelecidos e ativos.

É, portanto, imperativo que seja garantida a integridade deste ambiente na ESEC Rio Acre, teoricamente o primeiro a sofrer impactos antrópicos numa eventual investida contra os recursos florestais da unidade.

\section{CONCLUSÃO}

A avifauna registrada na ESEC Rio Acre revelou que a unidade se encontra ainda em perfeito estado de conservação. Com poucas exceções, praticamente todas as espécies de aves esperadas ou previamente documentadas para localidades próximas em países vizinhos foram registradas na ESEC Rio Acre, atestando que todos os sítios amostrados encontram-se em excelente estado de conservação. Igualmente importante foi o registro de grandes populações de espécies de alto valor cinegético, como Tinamus tao Temminck, 1815 (azulona) e Pauxi tuberosa (Spix, 1825) (mutum), intensamente perseguidas por caçadores e com estoques populacionais bastante reduzidos em outros pontos do Acre e Amazônia em geral (Silva \& Strahl, 1991; Sedaghatkish \& Brooks, 1999; González, 1999). Certamente, a ação de caçadores na ESEC Rio Acre tem sido tão esporádica que ainda não resultou em alterações populacionais significativas das principais espécies de aves caçadas na região, mas essa situação pode mudar rapidamente caso a unidade seja submetida a uma pressão antrópica maior.

A dinâmica da floresta com bambus e o seu papel na distribuição de várias espécies de aves e outros grupos de animais associados a esse tipo de vegetação (Conover, 1994; sensu Tobias et al., 2008) é uma questão ainda sem respostas claras, mas de extrema relevância para a preservação e eventual manejo destas espécies (Kratter, 1997; Silveira,
2005; Tobias et al., 2008). Em território brasileiro, a ESEC Rio Acre é a unidade de conservação com a mais rica avifauna associada a florestas com bambus (20 espécies no total), consistindo em um local ideal para estudos ecológicos de médio e longo prazos sobre essa associação.

\section{AGRADECIMENTOS}

Agradecemos ao Instituto Chico Mendes de Conservação da Biodiversidade (ICMBio), IBAMA, SOS Amazônia e World Wildlife Fund (WWF) - Brasil pela autorização (Licença 029/2005 - CG FAN) e apoio logístico e financeiro durante as expedições à ESEC Rio Acre. AA agradece ao Conselho Nacional de Desenvolvimento Científico e Tecnológico (CNPq) por uma bolsa de produtividade em pesquisa (auxílio n. 310593/2009-3).

\section{REFERÊNCIAS}

ALEIXO, A. \& E. GUILHERME, 2008. Avifauna da Estação Ecológica do Rio Acre, fronteira Brasil/Peru. Livro de Resumos do Congresso Brasileiro de Ornitologia 16: 305.

ALEIXO, A., E. GUILHERME \& K. J. ZIMMER, 2008. First records of Yellow-cheeked Becard Pachyramphus xanthogenys for Brazil, with comments on the validity of $P . x$. peruanus. Bulletin of the British Ornithological Club 128(4): 263-267.

ALEIXO, A., B. M. WHITNEY \& D. C. OREN, 2000. Range extensions of birds in southeastern Amazonia. The Wilson Bulletin 112(1): 137-142.

BATES, J. M., 1997. Distribution and geographic variation in three South American grassquits (Emberizinae, Tiaris). Ornithological Monographs 48: 91-110.

BATES, J. M., M. C. GARVIN, D. C. SCHMITT \& C. G. SCHMITT, 1989. Notes on bird distribution in northeastern Dpto. Santa Cruz, Bolivia, with 15 species new to Bolivia. Bulletin of the British Ornithological Club 109(4): 236-244.

CBRO, Comitê Brasileiro de Registros Ornitológicos, 2009. Lista das aves do Brasil. $8^{a}$ Edição. Disponível em: <http://www.cbro. org.br/CBRO/istabr.htm>. Acesso em: 7 setembro 2009.

CLEMENTS, J. F. \& N. SHANY, 2001. A field guide to the birds of Peru: 1-283. Ibis Publishing Company, Temecula, Califórnia.

CRACRAFT, J., 1985. Historical biogeography and patterns of differentiation within the South American avifauna: areas of endemism. Ornithological Monographs 36: 49-84.

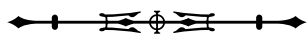


CONOVER, A., 1994. A new world comes to life, discovered in a stalk of bamboo. Smithsonian 25: 120-129.

DAVIS, T. J., C. FOX, L. SALINAS, G. BALLON \& C. ARANA, 1991. Annotated checklist of the birds of Cuzco Amazonico, Peru. Occasional Papers of the Museum of Natural History - The University of Kansas 114: 1-19.

FORRESTER, B. C., 1993. Birding Brazil - A check-list and site guide: 1-251. John Geddes, Irvane, Scotland.

GONZÁLEZ, J. A., 1999. Aves silvestres de importancia económica en el sector meridional de la reserva nacional Pacaya-Samiria (Loreto, Peru). In: T. G. FANG, O. L. MONTENEGRO \& R. E. BODMER (Eds.): Manejo y conservación de fauna silvestre en America Latina: 315-328. Editorial Instituto de Ecología, La Paz, Bolivia.

GUILHERME, E., 2001. Comunidade de Aves do Campus e Parque Zoobotânico da Universidade Federal do Acre, Brasil. Tangara 1(2): 57-73.

GUILHERME, E., 2004. Levantamento Ornitológico em Assis Brasil, Estado do Acre, na fronteira tri-nacional, Brasil, Peru e Bolívia. Livro de Resumos do Congresso Brasileiro de Ornitologia 22: 240.

GUILHERME, E., 2007. Levantamento preliminar da avifauna do complexo de florestas públicas estaduais do Mogno e dos rios Liberdade e Gregório, município de Tarauacá, estado do Acre, como subsídio para elaboração de seus planos de manejo. Atualidades Ornitológicas 136: 1-8.

GUILHERME, E. \& A. ALEIXO, 2008. Primeiros registros de Xiphorhynchus chunchotambo (Tschudi, 1884) (Dendrocolaptidae) no Brasil. Revista Brasileira de Ornitologia 16(1): 44-46.

GUILHERME, E. \& S. H. BORGES, 2008. Resultados ornitológicos de uma expedição a uma mancha de campinarana no alto Juruá, estado do Acre, Brasil. Livro de Resumos do Congresso Brasileiro de Ornitologia 16: 400

GUILHERME, E. \& S. M. DANTAS, 2008. Resultados ornitológicos de uma pesquisa no alto rio Purus, Estado do Acre, Brasil. Livro de Resumos do Congresso Brasileiro de Ornitologia 16: 401.

GUILHERME, E. \& M. P. D. SANTOS, 2009. Birds associated with bamboo forests in eastern Acre, Brazil. Bulletin of the British Ornithological Club 129(4): 229-240.

GYLDENSTOLPE, N., 1945. A contribution to the ornithology of northern Bolivia. Kungliga Svenska Vetenskapsakademiens Handlingar 23: 1-301.

GYLDENSTOLPE, N., 1951. The Ornithology of the rio Purús region in western Brazil. Arkiv För Zoologi 2(1): 1-320.

HAFFER, J., 1978. Distribution of Amazon forest birds. Bonner Zoologische Beiträge 29: 38-78.
HAFFER, J., 1987. Biogeography of Neotropical birds. In: T. C. WHITMORE \& G. T. PRANCE (Eds.): Biogeography and quaternary history in tropical America: 105-150. Claredon Press, Oxford.

HOYO, J. del., A. ELLIOTT \&J. SARGATAL (Eds.), 1992. Handbook of the birds of the world. Vol. 1. Ostrich to Ducks: 1- 696. Lynx Edicions, Barcelona.

HOYO, J. del., A. ELLIOTT \&J. SARGATAL (Eds.), 1997. Handbook of the birds of the world. Vol. 4. Sandgrouse to Cukoos: 1-679. Lynx Edicions, Barcelona.

HOYO, J. del., A. ELLIOTT \& J. SARGATAL (Eds.), 1999. Handbook of the birds of the world. Vol. 5. Barn-owls to Hummingbirds: 1-759. Lynx Edicions, Barcelona.

HOYO, J. del., A. ELLIOTT \& J. SARGATAL (Eds.), 2003. Handbook of the birds of the world. Vol. 8. Broadbills to Tapaculos: 1-845. Lynx Edicions, Barcelona.

HOYO, J. del., A. ELLIOTT \& D. A. CHRISTIE (Eds.), 2004. Handbook of the birds of the world. Vol. 9. Cotingas to Pipits and Wagtails: 1-863. Lynx Edicions, Barcelona.

ISA, Instituto Socioambiental, 2009. Unidades de Conservação na Amazônia. Disponível em: <http://www.socioambiental.org>. Acesso em: 1 maio 2009.

IUCN, 2008. Red list of threatened species. Disponível em: <http://www.redlist.org >. Acesso em: 20 maio 2009.

ISLER, M. L., P. R. ISLER \& B. M. WHITNEY, 2007. Species limits in antbirds (Thamnophilidae): the Warbling Antbird (Hypocnemis cantator) complex. The Auk 124(1): 11-28.

KRATTER, A. W., 1997. Bamboo specialization by Amazonian birds. Biotropica 29(1): 100-110.

LANE, D. F., G. P. SERVAT, T. VALQUI \& F. LAMBERT, 2007. A distinctive new species of Tyrant flycatcher (Passeriformes: Tyrannidae: Cnipodectes) from southweastern Peru. The Auk 124(3): 762-772.

LOWERY, G. H., JR. \&J. P. O'NEILL, 1966. A new Genus and Species of Cotinga from eastern Peru. The Auk 83(1): 1-9.

NOVAES, F. C., 1957. Contribuição à ornitologia do noroeste do Acre. Boletim do Museu Paraense Emílio Goeldi, Nova Série Zoologia 9: 1-30.

NOVAES, F. C., 1958. As aves e as comunidades bióticas no alto rio Juruá, Território do Acre. Boletim do Museu Paraense Emílio Goeldi, Nova Série Zoologia 14: 1-13.

NOVAES, F. C., 1978. Sobre algumas aves pouco conhecidas da Amazônia Brasileira II. Boletim do Museu Paraense Emílio Goeldi, série Zoologia 90: 1-15.

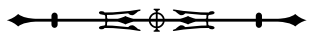


OREN, D. C. \& H. G. ALBUQUERQUE, 1991. Priority areas for new avian collections in Brazilian Amazonia. Goeldiana Zoologia 6: 1-11.

OREN, D. C. \&J. M. C. SILVA, 1987. Cherrie's Spinetail (Synallaxis cherriei Gyldenstolpe) (Aves: Furnariidae) in Carajás and Gorotire, Pará, Brazil. Boletim do Museu Paraense Emílio Goeldi, série Zoologia 3: 1-9.

PACHECO, J. F., 1995. New distributional records for some birds from várzea forest at Mamirauá Reserve, western Brazilian Amazonia. Ararajuba 3: 83-87

PARKER III, T. A., 1982. Observations of some unusual rainforest and marsh birds in southeastern Peru. The Willson Bulletin 94(4): 477-493.

PARKER III, T. A. \& J. V. REMSEN, JR., 1987. Fifty-two Amazonian bird species new to Bolivia. Bulletin of the British Ornithological Club 107(3): 94-107.

PARKER III, T. A., U. A. CASTILLO, M. GELL-MANN \& O. O. ROCHA, 1991. Records of new and unusual birds from northern Bolivia. Bulletin of the British Ornithological Club 111(3): 120-138.

PARKER III, T. A., P. K. DONAHUE \& T. S. SCHULENBERG, 1994. Birds of the Tambopata Reserve (Explorer's Inn Reserve). In: R. B. FOSTER, T. A. PARKER III, A. H. GENTRY, L. H. EMMONS, A. CHICCHÓN, T. SCHULENBERG, L. RODRÍGUEZ, G. LAMAS, H. ORTEGA, J. ICOCHEA, W. WUST, M. ROMO, J. A. CASTILLO, O. PHILLIPS, C. REYNEL, A. KRATTER, P. K. DONAHUE \& L. J. BARKLEY (Eds.): The Tambopata-Candamo Reserved zone of Southeastern Peru: A Biological Assessment: 106-124. Conservation Internacional, Washington.

PARKER III, T. A., D. F. STOTZ \& J. W. FITZPATRICK, 1997. Notes on avian bamboo specialists in southwestern amazonian Brazil. Ornithological Monographs 48: 543-547.

PETERS, J. L., 1979. Check-list of birds of the World: 8: 1-365. Museum of Comparative Zoology, Cambridge, Massachusetts.

PIERPONT, N. \& J. W. FITZPATRICK, 1983. Specific status and behavior of Cymbilaimus sanctaemariae, the bamboo antshrike, from southwestern Amazonia. The Auk 100(3): 645-652.

PINTO, O. M. O. \& E. A. CAMARGO, 1954. Resultados ornitológicos de uma expedição ao território do Acre pelo Departamento de Zoologia. Papéis Avulsos do Departamento de Zoologia 11(23): 371-418.

RASMUSSEN, D. T., J. REHG \& E. GUILHERME, 2005. Avifauna da Fazenda Experimental Catuaba: Uma pequena reserva florestal no leste do Estado do Acre, Brasil. In: P. M. DRUMOND (Org.): Fauna do Acre: 173-198. EDUFAC, Rio Branco.

REMSEN, J. V. JR. \& J. M. A. TRAYLOR, 1989. An annotated list of the birds of Bolivia: 1-79. Buteo Books, Vermillion, South Dakota.
RESTALL, R., C. RODNER \& M. LENTINO, 2006. Birds of Northern South America: An Identification Guide: 1: 1-880. Yale University Press, New Haven and London.

RIDGELY, R. S. \& G. TUDOR, 1994. The birds of South America: 2: 1-814. University of Texas Press, Austin.

SCHULENBERG, T. S., D. F. STOTZ, D. F. LANE, J. P. O'NEILL \& T. A. PARKER III, 2007. Birds of Peru: 1-656. Princeton University Press, New Jersey.

SEDAGHATKISH, G. \& D. M. BROOKS, 1999. Retraso evolutivo en los crácidos: Cantando para ser la cena del cazador. In: T. G. FANG, O. L. MONTENEGRO \& R. E. BODMER (Eds.): Manejo y conservación de fauna silvestre en America Latina: 335-340. Editorial Instituto de Ecologia, La Paz, Bolivia

SERVAT, G. P., 1996. An annotated list of birds of the Biolat Biological Station at Pakitza, Peru. In: D. E. WILSON \& A. SANDOVAL (Eds.): Manu: The biodiversity of southeastern Peru: 555-563. Smithsonian Institution, Washington.

SICK, H., 1997. Ornitologia brasileira: 1-862. Editora Nova Fronteira, Rio de Janeiro.

SILVA, J. L. \& S. D. STRAHL, 1991. Human impact on populations of chachalacas, guans, and curassows (Galliformes: Cracidae) in Venezuela. In: J. G. ROBINSON \& K. H. REDFORD (Eds.): Neotropical wildlife use and conservation: 37-52. University of Chicago Press, Chicago.

SILVA, J. M. C., A. B. RYLANDS \& G. A. B. FONSECA, 2005. O destino das áreas de endemismo da Amazônia. Megadiversidade 1(1): 124-131.

SILVEIRA, M., 2005. A floresta aberta com bambu do sudoeste da Amazônia: padrões e processos em múltiplas escalas: 1-153. EDUFAC, Rio Branco.

STOTZ, D. F., J. W. FITZPATRICK, T. A. PARKER III \& D. K. MOSKOVITS, 1996. Neotropical birds: ecology and conservation: 1-481. The University of Chicago Press, Chicago.

STOTZ, D. F., S. M. LANYON, T. S. SCHULENBERG, D. E. WILLARD, T. PETERSON \& J. W. FITZPATRICK, 1997. An avifaunal survey of two tropical forest localities on the middle rio ji-Parana, Rondônia, Brazil. Ornithological Monographs 48: 763-781.

TERBORGH, J., J. W. FITZPATRICK \& L. EMMONS, 1984. Annotated checklist of bird and mammal species of Cocha Cashu Biological Station, Manu National Park, Peru. Fieldiana Zoology 21: 1-29.

TERBORGH, J., S. K. ROBINSON, T. A. PARKER III, C. A. MUNN \& N. PIERPONT, 1990. Structure and organization of an Amazonian forest bird community. Ecological Monographs 60(2): 213-238.

TOBIAS, J. A. \& D. J. BRIGHTSMITH, 2007. Distribution, ecology and conservation status of the Blue-headed Macaw Primolius couloni. Biological Conservation 139(1-2): 126-138.

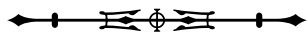


TOBIAS, J. A. \& N. SEDDON, 2007. Nine bird species new to Bolivia and notes on other significant records. Bulletin of the British Ornithological Club 127(1): 49-84.

TOBIAS, J. A., D. J. LEBBIN, A. ALEIXO, M. J. ANDERSEN, E. GUILHERME, P. A. HOSNER \& N. SEDDON, 2008. Distribution, behavior, and conservation status of the Rufous Twistwing (Cnipodectes superrufus). The Wilson Journal of Ornithology 120(1): 38-49.

ZIMMER, K. J., T. A. PARKER III, M. L. ISLER \& P. R. ISLER, 1997. Survey of a southern Amazonian avifauna: the Alta Floresta region, Mato Grosso, Brazil. Ornithological Monographs 48: 887-918.

WHITNEY, B. M. \& D. C. OREN, 2001a. Primeiro registro de Nannopsittaca dachilleae no Brasil. Nattereria 2: 26.

WHITNEY, B. M. \& D. C. OREN, 2001b. Documentação da ocorrência de Eubucco tucinkae no Brasil. Nattereria 2: 27.
WHITNEY, B. M. \& J. F. PACHECO, 2001. Evidência material para a presença de Vireo flavoviridis (Cassin, 1851) no Brasil. Nattereria 2: 36-37.

WHITTAKER, A. \& D. C. OREN, 1999. Important ornithological records from the Rio Juruá, western Amazonia, including twelve additions to the Brazilian avifauna. Bulletin of the British Ornithological Club 119(4): 235-260.

WHITTAKER, A., D. C. OREN, J. F. PACHECO, R. PARRINI \& J. C. MINNS, 2002. Aves registradas na Reserva extrativista do alto Juruá. In: M. C. CUNHA \& M. B. ALMEIDA (Orgs.): Enciclopédia da Floresta. O Alto Juruá: práticas e conhecimentos das populações: 81-99. Companhia das Letras, São Paulo.

Recebido: 11/09/2009

Aprovado: 06/12/2010

Responsabilidade editorial: Marinus Hoogmoed 
APÊNDICE. Lista sistemática das espécies de aves registradas ao longo de 180 horas de observação na ESEC Rio Acre, município de Assis Brasil, Acre, entre 13 e 24 de agosto de 2005 e 3 a 16 de fevereiro de 2006. Nomenclatura e nomes populares seguem CBRO (2009).

\begin{tabular}{l|c|c|c}
\multicolumn{1}{c|}{ Famílias e espécies } & FIT & $\mathrm{D}^{2}$ & $\mathrm{AB}^{3}$ \\
\hline TINAMIDAE (9) & & & \\
\hline Tinamus tao Temminck, 1815 *4 C & $\mathrm{P} / \mathrm{B}$ & $\mathrm{G}$ & $\mathrm{I}$ \\
\hline Tinamus major (Gmelin, 1789) & $\mathrm{A}, \mathrm{P} / \mathrm{B}$ & $\mathrm{E}(1)$ & $\mathrm{F}$ \\
\hline Crypturellus cinereus (Gmelin, 1789) & $\mathrm{A}$ & $\mathrm{G}$ & $\mathrm{I}$ \\
\hline Crypturellus soui (Hermann, 1783) & $\mathrm{A}$ & $\mathrm{G}$ & $\mathrm{R}$ \\
\hline Crypturellus obsoletus (Temminck, 1815)* ED & $\mathrm{P} / \mathrm{B}$ & $\mathrm{G}$ & $\mathrm{F}$ \\
\hline Crypturellus undulatus (Temminck, 1815) & $\mathrm{A}$ & $\mathrm{E}(1), \mathrm{G}$ & $\mathrm{Co}$ \\
\hline Crypturellus atrocapillus (Tschudi, 1844)* EN & $\mathrm{A}$ & $\mathrm{G}$ & $\mathrm{Co}$ \\
\hline Crypturellus variegatus (Gmelin, 1789) & $\mathrm{P} / \mathrm{B}$ & $\mathrm{O}$ & $\mathrm{R}$ \\
\hline Crypturellus bartletti (Sclater \& Salvin, 1873)* EN & $\mathrm{A}, \mathrm{P} / \mathrm{B}$ & $\mathrm{G}$ & $\mathrm{Co}$ \\
\hline ANATIDAE (1) & & & \\
\hline Cairina moschata (Linnaeus, 1758) & $\mathrm{R}$ & $\mathrm{O}$ & $\mathrm{I}$ \\
\hline CRACIDAE (4) & & & \\
\hline Ortalis guttata (Spix, 1825) & $\mathrm{A}$ & $\mathrm{O}$ & $\mathrm{Co}$ \\
\hline Penelope jacquacu Spix, 1825 & $\mathrm{A}, \mathrm{P} / \mathrm{B}$ & $\mathrm{O}$ & $\mathrm{I}$ \\
\hline Aburria cumanensis (Jacquin, 1784)* C & $\mathrm{A}$ & $\mathrm{O}$ & $\mathrm{I}$ \\
\hline Pauxi tuberosa (Spix, 1825)* C & $\mathrm{A}, \mathrm{P} / \mathrm{B}$ & $\mathrm{O}$ & $\mathrm{F}$ \\
\hline ODONTOPHORIDAE (1) & & & \\
\hline Odontophorus stellatus (Gould, 1843)* EN & $\mathrm{P} / \mathrm{B}$ & $\mathrm{E}(1), \mathrm{G}$ & $\mathrm{I}$ \\
\hline PHALACROCORACIDAE (1) & & & \\
\hline Phalacrocorax brasilianus (Gmelin, 1789) & $\mathrm{R}$ & $\mathrm{O}$ & $\mathrm{R}$ \\
\hline ARDEIDAE (5) & & & \\
\hline Butorides striata (Linnaeus, 1758) & $\mathrm{O}$ & $\mathrm{I}$ \\
\hline
\end{tabular}

1 Legenda dos principais tipos fitofisionômicos (FIT) reconhecidos pela avifauna (ver Tabela 1): A - Floresta aluvial de bambus e/ou palmeiras; $\mathrm{P} / \mathrm{B}$ - Floresta aberta de bambu e/ou palmeiras; $\mathbf{R}$ - ambiente ripário (praias e margens dos rios ).

2 Tipo de documentação (D) obtido para a espécie: E - espécime(s) testemunho coletado(s) e depositado(s) na Coleção Ornitológica do Museu Paraense Emílio Goeldi (MPEG) em Belém, Pará (números entre parênteses indicam a quantidade de indivíduos coletada para a espécie em questão); G - registro sonoro gravado em fita cassete e depositado no arquivo sonoro da Coleção Ornitológica do Museu Paraense Emílio Goeldi (MPEG) em Belém, Pará; O - espécie unicamente observada.

3 Legenda para abundância (AB): Comum (Co - visto ou ouvido durante todos os dias de amostragem por ambiente); Frequente (F visto ou ouvido em $50 \%$ dos dias de amostragem por ambiente); Incomum (I - visto ou ouvido em $25 \%$ dos dias de amostragem por ambiente) e Raro ( $\mathbf{R}$ - visto ou ouvido em apenas um dia de amostragem por ambiente).

4 Espécies em negrito e seguidas por um asterisco $(*)$ são aquelas de especial interesse para a conservação de acordo com os seguintes critérios: IUCN - espécies listadas como quase ameaçadas ou em perigo pela compilação mais recente da IUCN (2008); C - espécies de interesse cinegético (perseguidas por caçadores); ED - espécies cujos registros para a ESEC Rio Acre representam extensões significativas de distribuição para o sudoeste da Amazônia brasileira; EN - espécies endêmicas do Centro Amazônico de Endemismo Inambari (sensu Silva et al., 2005); M - espécies migratórias austrais e setentrionais; DL - espécies raras e de distribuição local na Amazônia ocidental; NR - espécies / táxons cujos registros para a ESEC Rio Acre representam ocorrências inéditas em território brasileiro.

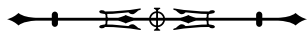


(Continua)

\begin{tabular}{|c|c|c|c|}
\hline Famílias e espécies & FIT & $\mathrm{D}$ & $A B$ \\
\hline Ardea cocoi Linnaeus, 1766 & $\mathrm{R}$ & O & I \\
\hline Pilherodius pileatus (Boddaert, 1783) & $\mathrm{R}$ & O & $\mathrm{R}$ \\
\hline Egretta thula (Molina, 1782) & $\mathrm{R}$ & O & I \\
\hline Cochlearius cochlearius (Linnaeus, 1766) & $\mathrm{R}$ & O & $\mathrm{R}$ \\
\hline \multicolumn{4}{|l|}{ THRESKIORNITHIDAE (1) } \\
\hline Mesembrinibis cayennensis (Gmelin, 1789) & $\mathrm{R}$ & O & I \\
\hline \multicolumn{4}{|l|}{ CATHARTIDAE (3) } \\
\hline Coragyps atratus & $\mathrm{R}$ & O & I \\
\hline Cathartes melambrotus Wetmore, 1964 & $\mathrm{R}$ & O & I \\
\hline Sarcoramphus papa (Linnaeus, 1758) & $\mathrm{R}$ & O & $\mathrm{R}$ \\
\hline \multicolumn{4}{|l|}{ ACCIPITRIDAE (7) } \\
\hline Harpagus bidentatus (Latham, 1790) & $\mathrm{P} / \mathrm{B}$ & $E(1), G$ & I \\
\hline Leucopternis schistaceus (Sundevall, 1851) & A & $\mathrm{O}$ & I \\
\hline Leucopternis albicollis (Latham, 1790) & $A, P / B$ & O & I \\
\hline Buteogallus urubitinga (Gmelin, 1788) & A & $E(1)$ & I \\
\hline Percnohierax leucorrhous (Quoy \& Gaimard, 1824)* ED & $\mathrm{A}, \mathrm{P} / \mathrm{B}$ & $\mathrm{O}$ & I \\
\hline Rupornis magnirostris (Gmelin, 1788) & A & $\mathrm{O}$ & $\mathrm{F}$ \\
\hline Spizaetus ornatus (Daudin, 1800) & $\mathrm{A}, \mathrm{P} / \mathrm{B}$ & G & I \\
\hline \multicolumn{4}{|l|}{ FALCONIDAE (6) } \\
\hline Daptrius ater Vieillot, 1816 & A & O & I \\
\hline Ibycter americanus (Boddaert, 1783) & $\mathrm{A}, \mathrm{P} / \mathrm{B}$ & O & $\mathrm{R}$ \\
\hline Micrastur ruficollis (Vieillot, 1817) & $\mathrm{A}, \mathrm{P} / \mathrm{B}$ & G & $\mathrm{F}$ \\
\hline Micrastur mirandollei (Schlegel, 1862) & $\mathrm{A}, \mathrm{P} / \mathrm{B}$ & O & $\mathrm{R}$ \\
\hline Micrastur semitorquatus (Vieillot, 1817) & $\mathrm{A}, \mathrm{P} / \mathrm{B}$ & G & I \\
\hline Falco rufigularis Daudin, 1800 & A & G & I \\
\hline \multicolumn{4}{|l|}{ PSOPHIIDAE (1) } \\
\hline Psophia leucoptera Spix, $1825 *$ EN & $\mathrm{P} / \mathrm{B}$ & $E(3)$ & $\mathrm{F}$ \\
\hline \multicolumn{4}{|l|}{ RALLIDAE (1) } \\
\hline Aramides cajanea (Statius Muller, 1776) & A & O & $\mathrm{R}$ \\
\hline \multicolumn{4}{|l|}{ HELIORNITHIDAE (1) } \\
\hline Heliornis fulica (Boddaert, 1783) & $\mathrm{R}$ & O & $\mathrm{R}$ \\
\hline \multicolumn{4}{|l|}{ EURYPYGIDAE (1) } \\
\hline Eurypyga helias (Pallas, 1781) & $\mathrm{R}$ & O & $\mathrm{R}$ \\
\hline \multicolumn{4}{|l|}{ CHARADRIIDAE (1) } \\
\hline Vanellus cayanus (Latham, 1790) & $\mathrm{R}$ & O & $\mathrm{F}$ \\
\hline \multicolumn{4}{|l|}{ SCOLOPACIDAE (2) } \\
\hline Tringa solitaria Wilson, $1813 * \mathrm{M}$ & $\mathrm{R}$ & 0 & $\mathrm{~F}$ \\
\hline
\end{tabular}


(Continua)

\begin{tabular}{|c|c|c|c|}
\hline Famílias e espécies & FIT & $\mathrm{D}$ & $A B$ \\
\hline Actitis macularius (Linnaeus, 1766)* M & $\mathrm{R}$ & $E(1)$ & $\mathrm{F}$ \\
\hline \multicolumn{4}{|l|}{ COLUMBIDAE (4) } \\
\hline Patagioenas plumbea (Vieillot, 1818) & $\mathrm{A}, \mathrm{P} / \mathrm{B}$ & G & $\mathrm{F}$ \\
\hline Patagioenas subvinacea (Lawrence, 1868) & $\mathrm{A}, \mathrm{P} / \mathrm{B}$ & $E(1), G$ & $\mathrm{~F}$ \\
\hline Leptotila rufaxilla (Richard \& Bernard, 1792) & A & $E(3), G$ & $\mathrm{~F}$ \\
\hline Geotrygon montana (Linnaeus, 1758) & $\mathrm{A}, \mathrm{P} / \mathrm{B}$ & $E(2), G$ & $\mathrm{R}$ \\
\hline \multicolumn{4}{|l|}{ PSITTACIDAE (18) } \\
\hline Ara ararauna (Linnaeus, 1758) & $\mathrm{A}, \mathrm{P} / \mathrm{B}$ & O & $\mathrm{R}$ \\
\hline Ara macao (Linnaeus, 1758) & $\mathrm{A}, \mathrm{P} / \mathrm{B}$ & O & I \\
\hline Ara chloropterus Gray, 1859 & $\mathrm{~A}, \mathrm{P} / \mathrm{B}$ & G & I \\
\hline Ara severus (Linnaeus, 1758) & A & O & $\mathrm{F}$ \\
\hline Orthopsittaca manilata (Boddaert, 1783) & A & G & I \\
\hline Primolius couloni (Sclater, 1876)* IUCN, EN & $\mathrm{A}, \mathrm{P} / \mathrm{B}$ & G & $\mathrm{F}$ \\
\hline Aratinga leucophthalma (Statius Muller, 1776) & A & G & $\mathrm{F}$ \\
\hline Aratinga weddellii (Deville, 1851) & A & O & I \\
\hline Pyrrhura roseifrons (Gray, 1859)* EN & $\mathrm{A}, \mathrm{P} / \mathrm{B}$ & O & I \\
\hline Pyrrhura rupicola (Tschudi, 1844)* EN & $\mathrm{A}, \mathrm{P} / \mathrm{B}$ & $E(1)$ & $\mathrm{F}$ \\
\hline Brotogeris cyanoptera (Pelzeln, 1870) & $\mathrm{A}, \mathrm{P} / \mathrm{B}$ & $E(3), G$ & Co \\
\hline Brotogeris sanctithomae (Statius Muller, 1776) & A & $E(1)$ & $\mathrm{F}$ \\
\hline Nannopsittaca dachilleae O’Neill, Munn \& Franke, 1991* IUCN & A & G & $\mathrm{F}$ \\
\hline Pionites leucogaster (Kuhl, 1820) & $\mathrm{A}, \mathrm{P} / \mathrm{B}$ & G & I \\
\hline Pyrilia barrabandi (Kuhl, 1820) & $\mathrm{A}, \mathrm{P} / \mathrm{B}$ & G & $\mathrm{F}$ \\
\hline Pionus menstruus (Linnaeus, 1766) & $\mathrm{A}, \mathrm{P} / \mathrm{B}$ & O & $\mathrm{F}$ \\
\hline Amazona ochrocephala (Gmelin, 1788) & A & G & $\mathrm{F}$ \\
\hline Amazona farinosa (Boddaert, 1783) & $\mathrm{A}, \mathrm{P} / \mathrm{B}$ & G & I \\
\hline \multicolumn{4}{|l|}{ OPISTHOCOMIDAE (1) } \\
\hline Opisthocomus hoazin (Statius Muller, 1776) & A & $E(1)$ & I \\
\hline \multicolumn{4}{|l|}{ CUCULIDAE (3) } \\
\hline Piaya cayana (Linnaeus, 1766) & $\mathrm{A}, \mathrm{P} / \mathrm{B}$ & G & I \\
\hline Dromococcyx phasianellus (Spix, 1824) & A & O & $\mathrm{R}$ \\
\hline Dromococcyx pavoninus Pelzeln, 1870 & A & $\mathrm{O}$ & I \\
\hline \multicolumn{4}{|l|}{ STRIGIDAE (7) } \\
\hline Megascops choliba (Vieillot, 1817) & A & $\mathrm{O}$ & $\mathrm{F}$ \\
\hline Megascops usta (Sclater, 1858) & $A, P / B$ & $E(1), G$ & I \\
\hline Pulsatrix perspicillata (Latham, 1790) & A & G & I \\
\hline Lophostrix cristata (Daudin, 1800) & A & $\mathrm{O}$ & I \\
\hline Strix virgata (Cassin, 1849) & A & $\mathrm{O}$ & I \\
\hline
\end{tabular}


(Continua)

\begin{tabular}{|c|c|c|c|}
\hline Famílias e espécies & FIT & $\mathrm{D}$ & $A B$ \\
\hline Glaucidium hardyi Vielliard, 1990 & $A, P / B$ & G & I \\
\hline Glaucidium brasilianum (Gmelin, 1788) & A & O & Co \\
\hline \multicolumn{4}{|l|}{ NYCTIBIIDAE (2) } \\
\hline Nyctibius grandis (Gmelin, 1789) & A & O & I \\
\hline Nyctibius griseus (Gmelin, 1789) & A & O & I \\
\hline \multicolumn{4}{|l|}{ CAPRIMULGIDAE (5) } \\
\hline Lurocalis semitorquatus (Gmelin, 1789) & $\mathrm{A}, \mathrm{P} / \mathrm{B}$ & G & $\mathrm{R}$ \\
\hline Nyctidromus albicollis (Gmelin, 1789) & A & O & $\mathrm{F}$ \\
\hline Nyctiphrynus ocellatus (Tschudi, 1844) & A & O & $\mathrm{F}$ \\
\hline Caprimulgus sericocaudatus (Cassin, 1849)* DL & $\mathrm{A}, \mathrm{P} / \mathrm{B}$ & O & I \\
\hline Hydropsalis climacocerca (Tschudi, 1844) & A & $E(1)$ & $\mathrm{R}$ \\
\hline \multicolumn{4}{|l|}{ APODIDAE (4) } \\
\hline Chaetura spinicaudus (Temminck, 1839) & $\mathrm{A}, \mathrm{P} / \mathrm{B}$ & O & $\mathrm{F}$ \\
\hline Chaetura cinereiventris Sclater, 1862 & $A, P / B$ & O & I \\
\hline Chaetura meridionalis Hellmayr, 1907* M & A & O & I \\
\hline Chaetura brachyura (Jardine, 1846) & A & O & $\mathrm{F}$ \\
\hline \multicolumn{4}{|l|}{ TROCHILIDAE (10) } \\
\hline Glaucis hirsutus (Gmelin, 1788) & A & $E(1)$ & I \\
\hline Threnetes leucurus (Linnaeus, 1766) & $\mathrm{A}, \mathrm{P} / \mathrm{B}$ & $E(1)$ & I \\
\hline Phaethornis ruber (Linnaeus, 1758) & $\mathrm{P} / \mathrm{B}$ & $E(1), G$ & I \\
\hline Phaethornis hispidus (Gould, 1846) & A & $E(6)$ & $\mathrm{F}$ \\
\hline Phaethornis malaris (Nordmann, 1835) & $\mathrm{A}, \mathrm{P} / \mathrm{B}$ & $E(1)$ & I \\
\hline Campylopterus largipennis (Boddaert, 1783) & A & $E(2)$ & $\mathrm{R}$ \\
\hline Thalurania furcata (Gmelin, 1788) & $\mathrm{A}, \mathrm{P} / \mathrm{B}$ & $E(1)$ & $\mathrm{F}$ \\
\hline Florisuga mellivora (Linnaeus, 1758) & A & $E(1)$ & $\mathrm{R}$ \\
\hline Amazilia lactea bartletti (Sclater \& Salvin, 1866)* ED & $\mathrm{R}$ & O & I \\
\hline Heliodoxa aurescens (Gould, 1846) & $\mathrm{P} / \mathrm{B}$ & G & I \\
\hline \multicolumn{4}{|l|}{ TROGONIDAE (6) } \\
\hline Trogon melanurus Swainson, 1838 & $\mathrm{~A}, \mathrm{P} / \mathrm{B}$ & $E(1), G$ & $\mathrm{~F}$ \\
\hline Trogon viridis Linnaeus, 1766 & $\mathrm{P} / \mathrm{B}$ & $E(1), G$ & I \\
\hline Trogon ramonianus Deville \& DesMurs, 1849 & $\mathrm{P} / \mathrm{B}$ & $E(1)$ & $\mathrm{R}$ \\
\hline Trogon curucui Linnaeus, 1766 & A & $E(1)$ & I \\
\hline Trogon rufus Gmelin, 1788 & $\mathrm{P} / \mathrm{B}$ & G & $\mathrm{R}$ \\
\hline Trogon collaris Vieillot, 1817 & A & O & I \\
\hline \multicolumn{4}{|l|}{ ALCEDINIDAE (4) } \\
\hline Ceryle torquatus (Linnaeus, 1766) & $\mathrm{R}$ & $\mathrm{O}$ & $\mathrm{R}$ \\
\hline Chloroceryle amazona (Latham, 1790) & $\mathrm{R}$ & 0 & $\mathrm{~F}$ \\
\hline
\end{tabular}


(Continua)

\begin{tabular}{|c|c|c|c|}
\hline Famílias e espécies & FIT & D & $A B$ \\
\hline Chlorocenyle americana (Gmelin, 1788) & $\mathrm{R}$ & O & $\mathrm{F}$ \\
\hline Chloroceryle aenea (Pallas, 1764) & A & $E(1)$ & $\mathrm{R}$ \\
\hline \multicolumn{4}{|l|}{ MOMOTIDAE (3) } \\
\hline Electron platyrhynchum (Leadbeater, 1829) & A, P/B & $E(1), G$ & I \\
\hline Baryphthengus martii (Spix, 1824) & $\mathrm{A}, \mathrm{P} / \mathrm{B}$ & $E(1), G$ & $\mathrm{R}$ \\
\hline Momotus momota (Linnaeus, 1766) & $\mathrm{A}, \mathrm{P} / \mathrm{B}$ & $E(2), G$ & $\mathrm{~F}$ \\
\hline \multicolumn{4}{|l|}{ GALBULIDAE (4) } \\
\hline Galbalcyrhynchus purusianus Goeldi, 1904* EN & A & $E(2), G$ & $\mathrm{R}$ \\
\hline Brachygalba albogularis (Spix, 1824)* EN & A & $E(4)$ & Co \\
\hline Galbula cyanescens Deville, 1849* EN & $\mathrm{A}, \mathrm{P} / \mathrm{B}$ & $E(3), G$ & Co \\
\hline Galbula dea (Linnaeus, 1758) & $\mathrm{P} / \mathrm{B}$ & G & $\mathrm{R}$ \\
\hline \multicolumn{4}{|l|}{ BUCCONIDAE (11) } \\
\hline Notharchus hyperrhynchus (Sclater, 1856) & $\mathrm{A}, \mathrm{P} / \mathrm{B}$ & G & $\mathrm{I}$ \\
\hline Bucco macrodactylus (Spix, 1824) & A & G & $\mathrm{F}$ \\
\hline Bucco capensis Linnaeus, 1766 & $\mathrm{P} / \mathrm{B}$ & G & $\mathrm{R}$ \\
\hline Nystalus striolatus (Pelzeln, 1856) & A & G & I \\
\hline Malacoptila semicincta Todd, $1925 * \mathrm{EN}$ & $\mathrm{P} / \mathrm{B}$ & $E(2), G$ & $\mathrm{R}$ \\
\hline Nonnula sclateri Hellmayr, 1907* EN & $\mathrm{P} / \mathrm{B}$ & O & $\mathrm{R}$ \\
\hline Nonnula ruficapilla (Tschudi, 1844) & A & $E(1)$ & $\mathrm{R}$ \\
\hline Monasa nigrifrons (Spix, 1824) & A & $E(1)$ & $\mathrm{Co}$ \\
\hline Monasa morphoeus (Hahn \& Küster, 1823) & $\mathrm{P} / \mathrm{B}$ & $E(1)$ & $\mathrm{R}$ \\
\hline Monasa flavirostris Strickland, $1850 * \mathrm{DL}$ & $\mathrm{P} / \mathrm{B}$ & G & I \\
\hline Chelidoptera tenebrosa (Pallas, 1782) & A & O & $\mathrm{F}$ \\
\hline \multicolumn{4}{|l|}{ CAPITONIDAE (3) } \\
\hline Capito auratus (Dumont, 1816) & $\mathrm{P} / \mathrm{B}$ & $\mathrm{O}$ & I \\
\hline Eubucco richardsoni (Gray, 1846) & A & O & I \\
\hline Eubucco tucinkae (Seilern, 1913)* EN & A & $\mathrm{O}$ & $\mathrm{R}$ \\
\hline \multicolumn{4}{|l|}{ RAMPHASTIDAE (7) } \\
\hline Ramphastos tucanus Linnaeus, 1758 & $\mathrm{~A}, \mathrm{P} / \mathrm{B}$ & $E(2), G$ & $\mathrm{~F}$ \\
\hline Ramphastos vitellinus Lichtenstein, 1823 & $\mathrm{~A}, \mathrm{P} / \mathrm{B}$ & O & $\mathrm{F}$ \\
\hline Aulacorhynchus atrogularis (Sturm \& Sturm, 1841)* ED & A & O & I \\
\hline Pteroglossus inscriptus Swainson, 1822 & A & O & I \\
\hline Pteroglossus mariae Gould, 1854* EN & $\mathrm{A}, \mathrm{P} / \mathrm{B}$ & $E(2)$ & $\mathrm{I}$ \\
\hline Pteroglossus castanotis Gould, 1834 & A & G & I \\
\hline Pteroglossus beauharnaesii Wagler, $1832 * \mathrm{EN}$ & $\mathrm{P} / \mathrm{B}$ & G & $\mathrm{F}$ \\
\hline \multicolumn{4}{|l|}{ PICIDAE (14) } \\
\hline Picumnus rufiventris Bonaparte, 1838 * DL & $\mathrm{P} / \mathrm{B}$ & G & $\mathrm{F}$ \\
\hline
\end{tabular}


(Continua)

\begin{tabular}{|c|c|c|c|}
\hline Famílias e espécies & FIT & $\mathrm{D}$ & $A B$ \\
\hline Melanerpes cruentatus (Boddaert, 1783) & $A, P / B$ & G & $\mathrm{F}$ \\
\hline Veniliornis passerinus (Linnaeus, 1766) & A & 0 & $\mathrm{~F}$ \\
\hline Veniliornis affinis (Swainson, 1821) & $A, P / B$ & $E(1), G$ & I \\
\hline Piculus leucolaemus (Natterer \& Malherbe, 1845) & $\mathrm{P} / \mathrm{B}$ & G & $\mathrm{R}$ \\
\hline Piculus flavigula (Boddaert, 1783) & $A, P / B$ & G & I \\
\hline Piculus chrysochloros (Vieillot, 1818) & A & G & I \\
\hline Celeus grammicus (Natterer \& Malherbe, 1845) & $A, P / B$ & ○ & $\mathrm{R}$ \\
\hline Celeus flavus (Statius Muller, 1776) & $A, P / B$ & ○ & I \\
\hline Celeus spectabilis Sclater \& Salvin, 1880* DL & A & O & $\mathrm{F}$ \\
\hline Celeus torquatus (Boddaert, 1783) & $\mathrm{P} / \mathrm{B}$ & $E(1)$ & I \\
\hline Dryocopus lineatus (Linnaeus, 1766) & A & O & I \\
\hline Campephilus rubricollis (Boddaert, 1783) & $A, P / B$ & $E(2)$ & $\mathrm{F}$ \\
\hline Campephilus melanoleucos (Gmelin, 1788) & A & $E(3)$ & I \\
\hline \multicolumn{4}{|l|}{ THAMNOPHILIDAE (40) } \\
\hline Cymbilaimus lineatus (Leach, 1814) & $\mathrm{P} / \mathrm{B}$ & $E(1), G$ & I \\
\hline Cymbilaimus sanctaemariae Gyldenstolpe, 1941 * DL & A & $E(2), G$ & Co \\
\hline Taraba major (Vieillot, 1816) & A & $E(1), G$ & $\mathrm{~F}$ \\
\hline Thamnophilus aethiops Sclater, 1858 & $\mathrm{P} / \mathrm{B}$ & $E(1), G$ & I \\
\hline Thamnophilus schistaceus d'Orbigny, 1835 & $\mathrm{~A}, \mathrm{P} / \mathrm{B}$ & $E(2), G$ & $\mathrm{~F}$ \\
\hline Thamnophilus murinus Sclater \& Salvin, 1868 & $\mathrm{P} / \mathrm{B}$ & O & I \\
\hline Thamnomanes schistogynus Hellmayr, 1911* EN & $\mathrm{A}, \mathrm{P} / \mathrm{B}$ & $E(4), G$ & I \\
\hline Epinecrophylla leucophthalma (Pelzeln, 1868) & $\mathrm{P} / \mathrm{B}$ & O & I \\
\hline Epinecrophylla haematonota (Sclater, 1857) & $\mathrm{P} / \mathrm{B}$ & O & I \\
\hline Epinecrophylla ornata (Sclater, 1853) & $\mathrm{A}, \mathrm{P} / \mathrm{B}$ & $E(1), G$ & $\mathrm{~F}$ \\
\hline Myrmotherula brachyura (Hermann, 1783) & $\mathrm{A}, \mathrm{P} / \mathrm{B}$ & G & $\mathrm{F}$ \\
\hline Myrmotherula sclateri Snethlage, 1912 & $A, P / B$ & G & $\mathrm{F}$ \\
\hline Myrmotherula hauxwelli (Sclater, 1857) & $\mathrm{P} / \mathrm{B}$ & G & I \\
\hline Myrmotherula axillaris (Vieillot, 1817) & $\mathrm{A}, \mathrm{P} / \mathrm{B}$ & $E(4), G$ & $\mathrm{~F}$ \\
\hline Myrmotherula longipennis Pelzeln, 1868 & $\mathrm{P} / \mathrm{B}$ & $\mathrm{O}$ & I \\
\hline Myrmotherula iheringi Snethlage, 1914 & $\mathrm{~A}, \mathrm{P} / \mathrm{B}$ & G & I \\
\hline Myrmotherula menetriesii (d'Orbigny, 1837) & $\mathrm{P} / \mathrm{B}$ & G & I \\
\hline Dichrozona cincta (Pelzeln, 1868) & $\mathrm{P} / \mathrm{B}$ & $E(1)$ & $\mathrm{R}$ \\
\hline Herpsilochmus rufimarginatus (Temminck, 1822)* ED & $\mathrm{P} / \mathrm{B}$ & $E(2), G$ & $\mathrm{~F}$ \\
\hline Microrhopias quixensis (Cornalia, 1849) & $A, P / B$ & $E(1), G$ & I \\
\hline Drymophila devillei (Menegaux \& Hellmayr, 1906)* DL & A & G & I \\
\hline Cercomacra cinerascens (Sclater, 1857) & $\mathrm{A}, \mathrm{P} / \mathrm{B}$ & $E(1), G$ & I \\
\hline Cercomacra nigrescens (Cabanis \& Heine, 1859) & A & 0 & I \\
\hline
\end{tabular}


(Continua)

\begin{tabular}{|c|c|c|c|}
\hline Famílias e espécies & FIT & $\mathrm{D}$ & $A B$ \\
\hline Cercomacra serva (Sclater, 1858)* EN & A & O & $\mathrm{R}$ \\
\hline Cercomacra manu Fitzpatrick \& Willard, 1990* DL & A & G & $\mathrm{F}$ \\
\hline Myrmoborus leucophrys (Tschudi, 1844) & A & $E(3), G$ & $\mathrm{~F}$ \\
\hline Myrmoborus myotherinus (Spix, 1825) & $\mathrm{P} / \mathrm{B}$ & $E(3), G$ & I \\
\hline Hypocnemis subflava collinsi Cherrie, 1916* NR & A & $E(2), G$ & I \\
\hline Sclateria naevia (Gmelin, 1788) & A & O & I \\
\hline Percnostola lophotes Hellmayr \& Seilern, 1914* EN & A & G & Co \\
\hline Myrmeciza hemimelaena Sclater, 1857 & $\mathrm{P} / \mathrm{B}$ & $E(2), G$ & $\mathrm{~F}$ \\
\hline Myrmeciza atrothorax (Boddaert, 1783) & A & O & $\mathrm{F}$ \\
\hline Myrmeciza goeldii (Snethlage, 1908)* EN & A & $E(4), G$ & Co \\
\hline Myrmeciza hyperythra (Sclater, 1855)* EN & A & $E(3), G$ & $\mathrm{R}$ \\
\hline Myrmeciza fortis (Sclater \& Salvin, 1868)* EN & $\mathrm{P} / \mathrm{B}$ & $E(1), G$ & $\mathrm{R}$ \\
\hline Gymnopithys salvini (Berlepsch, 1901) & $\mathrm{P} / \mathrm{B}$ & $E(6), G$ & I \\
\hline Rhegmatorhina melanosticta (Sclater \& Salvin, 1880) & $\mathrm{A}, \mathrm{P} / \mathrm{B}$ & O & I \\
\hline Hylophylax naevius (Gmelin, 1789) & $\mathrm{A}, \mathrm{P} / \mathrm{B}$ & $E(5)$ & I \\
\hline Willisornis poecilinotus (Cabanis, 1847) & $\mathrm{P} / \mathrm{B}$ & $E(4), G$ & I \\
\hline Phlegopsis nigromaculata (d'Orbigny \& Lafresnaye, 1837) & $\mathrm{A}, \mathrm{P} / \mathrm{B}$ & $E(2), G$ & I \\
\hline \multicolumn{4}{|l|}{ CONOPOPHAGIDAE (2) } \\
\hline$[\text { Conopophaga aurita (Gmelin, 1789)] }]^{5}$ & $\mathrm{P} / \mathrm{B}$ & G & $\mathrm{R}$ \\
\hline Conopophaga peruviana (Des Murs, 1856) & A & E & $\mathrm{R}$ \\
\hline \multicolumn{4}{|l|}{ GRALLARIIDAE (2) } \\
\hline Hylopezus berlepschi (Hellmayr, 1903) & A & O & $\mathrm{R}$ \\
\hline Myrmothera campanisona (Hermann, 1783) & $\mathrm{P} / \mathrm{B}$ & G & I \\
\hline \multicolumn{4}{|l|}{ RHINOCRYPTIDAE (1) } \\
\hline Liosceles thoracicus (Sclater, 1865) & $\mathrm{P} / \mathrm{B}$ & G & $\mathrm{R}$ \\
\hline \multicolumn{4}{|l|}{ FORMICARIIDAE (3) } \\
\hline Formicarius colma Boddaert, 1783 & $\mathrm{P} / \mathrm{B}$ & $E(1)$ & $\mathrm{R}$ \\
\hline Formicarius analis (d'Orbigny \& Lafresnaye, 1837) & $\mathrm{A}, \mathrm{P} / \mathrm{B}$ & $E(1), G$ & I \\
\hline Formicarius rufifrons Blake, $1957^{*}$ IUCN & A & $\mathrm{O}$ & $R$ \\
\hline \multicolumn{4}{|l|}{ SCLERURIDAE (3) } \\
\hline Sclerurus mexicanus Sclater, 1857 & A & $E(2)$ & $\mathrm{R}$ \\
\hline Sclerurus caudacutus (Vieillot, 1816) & $\mathrm{P} / \mathrm{B}$ & $E(1), G$ & I \\
\hline Sclerurus albigularis Sclater \& Salvin, 1869* DL & $\mathrm{P} / \mathrm{B}$ & $E(2), G$ & $\mathrm{I}$ \\
\hline DENDROCOLAPTIDAE (16) & & & \\
\hline
\end{tabular}

5 Nomes científicos entre colchetes indicam que a identificação da espécie em questão não foi obtida com o devido grau de certeza desejado, necessitando de futura confirmação.

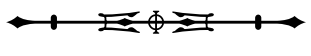


(Continua)

\begin{tabular}{|c|c|c|c|}
\hline Famílias e espécies & FIT & $\mathrm{D}$ & $A B$ \\
\hline Dendrocincla fuliginosa (Vieillot, 1818) & A & $E(7), G$ & Co \\
\hline Dendrocincla merula (Lichtenstein, 1829) & $\mathrm{P} / \mathrm{B}$ & $E(1)$ & $\mathrm{R}$ \\
\hline Deconychura longicauda (Pelzeln, 1868) & $\mathrm{P} / \mathrm{B}$ & O & I \\
\hline Deconychura stictolaema (Pelzeln, 1868) & $\mathrm{P} / \mathrm{B}$ & O & $\mathrm{R}$ \\
\hline Sittasomus griseicapillus (Vieillot, 1818) & $\mathrm{A}, \mathrm{P} / \mathrm{B}$ & $E(7), G$ & Co \\
\hline Glyphorynchus spirurus albigularis (Vieillot, 1819)* NR & A & $E(3)$ & $\mathrm{R}$ \\
\hline Nasica longirostris (Vieillot, 1818) & A & O & I \\
\hline Dendrexetastes rufigula (Lesson, 1844) & $\mathrm{A}, \mathrm{P} / \mathrm{B}$ & $E(2)$ & $\mathrm{F}$ \\
\hline [Hylexetastes stresemanni Snethlage, 1925] & A & O & $\mathrm{R}$ \\
\hline Xiphocolaptes promeropirhynchus (Lesson, 1840) & $\mathrm{A}, \mathrm{P} / \mathrm{B}$ & G & I \\
\hline Dendrocolaptes certhia (Boddaert, 1783) & $\mathrm{A}, \mathrm{P} / \mathrm{B}$ & $E(4), G$ & $\mathrm{~F}$ \\
\hline Dendrocolaptes picumnus Lichtenstein, 1820 & A & $E(1), G$ & I \\
\hline Xiphorhynchus chunchotambo Tschudi, 1844* NR & A & $E(3), G$ & I \\
\hline Xiphorhynchus guttatus (Lichtenstein, 1820) & $\mathrm{A}, \mathrm{P} / \mathrm{B}$ & $E(6), G$ & $\mathrm{~F}$ \\
\hline Lepidocolaptes albolineatus (Lafresnaye, 1845) & $\mathrm{A}, \mathrm{P} / \mathrm{B}$ & O & $\mathrm{I}$ \\
\hline Campylorhamphus trochilirostris (Lichtenstein, 1820) & $\mathrm{A}, \mathrm{P} / \mathrm{B}$ & $E(5), G$ & I \\
\hline \multicolumn{4}{|l|}{ FURNARIIDAE (19) } \\
\hline Furnarius leucopus Swainson, 1838 & A & $E(3)$ & Co \\
\hline Synallaxis sp. ${ }^{6}$ & A & O & $\mathrm{R}$ \\
\hline Synallaxis rutilans Temminck, 1823 & $\mathrm{P} / \mathrm{B}$ & $\mathrm{O}$ & I \\
\hline Synallaxis cherriei Gyldenstolpe, 1930* IUCN & $\mathrm{A}, \mathrm{P} / \mathrm{B}$ & O & $\mathrm{I}$ \\
\hline Metopothrix aurantiaca Sclater \& Salvin, 1866* DL & A & $E(2)$ & $\mathrm{R}$ \\
\hline Simoxenops ucayalae (Chapman, 1928)* IUCN & $A, P / B$ & $E(1)$ & I \\
\hline Ancistrops strigilatus (Spix, 1825) & $\mathrm{P} / \mathrm{B}$ & $E(1), G$ & I \\
\hline Hyloctistes subulatus (Spix, 1824) & $\mathrm{P} / \mathrm{B}$ & $E(2)$ & I \\
\hline Philydor ruficaudatum (d'Orbigny \& Lafresnaye, 1838) & $\mathrm{A}, \mathrm{P} / \mathrm{B}$ & $E(1)$ & I \\
\hline Philydor enythropterum (Sclater, 1856) & A & O & I \\
\hline Philydor rufum bolivianum (Vieillot, 1818)* NR & A & $E(1)$ & $\mathrm{R}$ \\
\hline Anabazenops dorsalis (Sclater \& Salvin, 1880)* DL & $\mathrm{A}, \mathrm{P} / \mathrm{B}$ & O & $\mathrm{R}$ \\
\hline Automolus ochrolaemus (Tschudi, 1844) & $\mathrm{A}, \mathrm{P} / \mathrm{B}$ & $E(8), G$ & I \\
\hline Automolus infuscatus (Sclater, 1856) & A & O & $\mathrm{R}$ \\
\hline Automolus melanopezus (Sclater, 1858)* DL & $\mathrm{P} / \mathrm{B}$ & $E(1)$ & $\mathrm{R}$ \\
\hline Automolus rubiginosus (Sclater, 1857)* DL & $\mathrm{P} / \mathrm{B}$ & $E(2), G$ & $\mathrm{R}$ \\
\hline Automolus rufipileatus (Pelzeln, 1859) & A & $E(1)$ & I \\
\hline
\end{tabular}

6 As letras sp. seguidas por um gênero indicam a presença de uma espécie distinta para o gênero em questão na área de estudo, mas cuja identificação em nível específico não pode ainda ser feita com segurança.

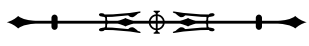


(Continua)

\begin{tabular}{|c|c|c|c|}
\hline Famílias e espécies & FIT & $\mathrm{D}$ & $A B$ \\
\hline Xenops tenuirostris Pelzeln, 1859 & $\mathrm{P} / \mathrm{B}$ & $\mathrm{O}$ & $\mathrm{I}$ \\
\hline Xenops minutus (Sparrman, 1788) & A & $E(3)$ & I \\
\hline \multicolumn{4}{|l|}{ TYRANNIDAE (47) } \\
\hline Mionectes oleagineus (Lichtenstein, 1823) & $\mathrm{P} / \mathrm{B}$ & O & $\mathrm{R}$ \\
\hline Leptopogon amaurocephalus Tschudi, 1846 & $\mathrm{~A}, \mathrm{P} / \mathrm{B}$ & $E(3)$ & $\mathrm{F}$ \\
\hline Corythopis torquatus (Tschudi, 1844) & $\mathrm{P} / \mathrm{B}$ & $E(2), G$ & I \\
\hline Lophotriccus eulophotes Todd, 1925* EN & $\mathrm{A}, \mathrm{P} / \mathrm{B}$ & $E(2), G$ & I \\
\hline Hemitriccus flammulatus Berlepsch, 1901* DL & $A, P / B$ & $E(3), G$ & I \\
\hline Myiornis ecaudatus (d'Orbigny \& Lafresnaye, 1837) & $\mathrm{P} / \mathrm{B}$ & $\mathrm{O}$ & $\mathrm{F}$ \\
\hline Todirostrum maculatum (Desmarest, 1806) & A & O & $\mathrm{R}$ \\
\hline Todirostrum chrysocrotaphum Strickland, 1850 & A & O & I \\
\hline Tyrannulus elatus (Latham, 1790) & $\mathrm{P} / \mathrm{B}$ & O & $\mathrm{R}$ \\
\hline Myiopagis gaimardii (d'Orbigny, 1839) & $\mathrm{A}, \mathrm{P} / \mathrm{B}$ & G & I \\
\hline Myiopagis caniceps (Swainson, 1835) & $\mathrm{A}, \mathrm{P} / \mathrm{B}$ & G & I \\
\hline Ornithion inerme Hartlaub, 1853 & $\mathrm{~A}, \mathrm{P} / \mathrm{B}$ & O & $\mathrm{R}$ \\
\hline Zimmerius gracilipes (Sclater \& Salvin, 1868) & $\mathrm{A}, \mathrm{P} / \mathrm{B}$ & G & I \\
\hline Cnipodectes superrufus Lane, Servat, Valqui \& Lambert, 2007* EN & A & O & I \\
\hline Rhynchocyclus olivaceus (Temminck, 1820) & A & $E(2)$ & I \\
\hline Tolmomyias assimilis (Pelzeln, 1868) & $A, P / B$ & G & I \\
\hline Tolmomyias poliocephalus (Taczanowski, 1884) & $\mathrm{A}, \mathrm{P} / \mathrm{B}$ & G & I \\
\hline Tolmomyias flaviventris (Wied, 1831) & A & G & I \\
\hline Platyrinchus platyrhynchos (Gmelin, 1788) & $\mathrm{P} / \mathrm{B}$ & $E(1), G$ & I \\
\hline Onychorhynchus coronatus (Statius Muller, 1776) & A & $E(2)$ & $\mathrm{R}$ \\
\hline Myiophobus fasciatus (Statius Muller, 1776) & A & 0 & I \\
\hline Terenotriccus erythrurus (Cabanis, 1847) & $\mathrm{A}, \mathrm{P} / \mathrm{B}$ & $\mathrm{O}$ & I \\
\hline Lathrotriccus euleri (Cabanis, 1868) & A & $E(1)$ & I \\
\hline Contopus virens (Linnaeus, 1766)* M & $\mathrm{A}, \mathrm{P} / \mathrm{B}$ & G & $\mathrm{F}$ \\
\hline Pyrocephalus rubinus (Boddaert, 1783)* M & A & $E(1)$ & $\mathrm{F}$ \\
\hline Ochthornis littoralis (Pelzeln, 1868) & A & $E(1)$ & $\mathrm{F}$ \\
\hline Colonia colonus (Vieillot, 1818) & A & $\mathrm{O}$ & I \\
\hline Legatus leucophaius (Vieillot, 1818) & $\mathrm{A}, \mathrm{P} / \mathrm{B}$ & O & I \\
\hline Myiozetetes similis (Spix, 1825) & A & $E(1)$ & I \\
\hline Myiozetetes granadensis Lawrence, $1862 * \mathrm{DL}$ & A & $E(1), G$ & I \\
\hline Myiozetetes luteiventris (Sclater, 1858) & A & O & I \\
\hline Pitangus sulphuratus (Linnaeus, 1766) & A & $\mathrm{O}$ & I \\
\hline Conopias trivirgatus (Wied, 1831) & $\mathrm{P} / \mathrm{B}$ & G & I \\
\hline Myiodynastes maculatus (Statius Muller, 1776) & $A, P / B$ & O & I \\
\hline
\end{tabular}


(Continua)

\begin{tabular}{|c|c|c|c|}
\hline Famílias e espécies & FIT & $\mathrm{D}$ & $A B$ \\
\hline Megarynchus pitangua (Linnaeus, 1766) & A & O & I \\
\hline Griseotyrannus aurantioatrocristatus (d'Orbigny \& Lafresnaye, 1837) & A & $\mathrm{O}$ & $\mathrm{R}$ \\
\hline Tyrannus melancholicus Vieillot, 1819 & A & O & I \\
\hline Tyrannus savana Vieillot, 1808 & A & O & I* \\
\hline Tyrannus tyrannus (Linnaeus, 1766)* M & A & O & I* \\
\hline Rhytipterna simplex (Lichtenstein, 1823) & $\mathrm{A}, \mathrm{P} / \mathrm{B}$ & $E(2)$ & $\mathrm{F}$ \\
\hline Sirystes sibilator (Vieillot, 1818) & $A, P / B$ & $\mathrm{O}$ & $\mathrm{F}$ \\
\hline Myiarchus tuberculifer (d'Orbigny \& Lafresnaye, 1837) & $\mathrm{A}, \mathrm{P} / \mathrm{B}$ & $E(2), G$ & $\mathrm{~F}$ \\
\hline Myiarchus ferox (Gmelin, 1789) & A & O & I \\
\hline Ramphotrigon megacephalum (Swainson, 1835) & $\mathrm{A}, \mathrm{P} / \mathrm{B}$ & G & I \\
\hline Ramphotrigon fuscicauda Chapman, $1925 *$ DL & $\mathrm{P} / \mathrm{B}$ & $E(3), G$ & $\mathrm{~F}$ \\
\hline Attila bolivianus Lafresnaye, 1848 & A & $E(2)$ & $\mathrm{F}$ \\
\hline Attila spadiceus (Gmelin, 1789) & $\mathrm{P} / \mathrm{B}$ & $E(2), G$ & I \\
\hline \multicolumn{4}{|l|}{ COTINGIDAE (4) } \\
\hline Lipaugus vociferans (Wied, 1820) & $\mathrm{P} / \mathrm{B}$ & O & $\mathrm{R}$ \\
\hline Conioptilon mcilhennyi Lowery \& O’Neill, 1966* EN & $\mathrm{A}, \mathrm{P} / \mathrm{B}$ & $E(1), G$ & $\mathrm{~F}$ \\
\hline Gymnoderus foetidus (Linnaeus, 1758) & A & O & $\mathrm{F}$ \\
\hline Querula purpurata (Statius Muller, 1776) & $\mathrm{A}, \mathrm{P} / \mathrm{B}$ & G & I \\
\hline \multicolumn{4}{|l|}{ PIPRIDAE (5) } \\
\hline Neopelma sulphureiventer (Hellmayr, 1903)* DL & A & $E(3)$ & I \\
\hline Piprites chloris (Temminck, 1822) & $\mathrm{A}, \mathrm{P} / \mathrm{B}$ & O & I \\
\hline Lepidothrix coronata (Spix, 1825) & $\mathrm{P} / \mathrm{B}$ & O & I \\
\hline Pipra fasciicauda Hellmayr, 1906 & A & $E(6)$ & $\mathrm{F}$ \\
\hline Pipra chloromeros Tschudi, $1844 * \mathrm{DL}$ & $\mathrm{A}, \mathrm{P} / \mathrm{B}$ & $E(2)$ & I \\
\hline \multicolumn{4}{|l|}{ TITYRIDAE (7) } \\
\hline Tityra inquisitor (Lichtenstein, 1823) & $\mathrm{P} / \mathrm{B}$ & O & $\mathrm{R}$ \\
\hline Tityra cayana (Linnaeus, 1766) & A & O & I \\
\hline Tityra semifasciata (Spix, 1825) & $\mathrm{A}, \mathrm{P} / \mathrm{B}$ & G & I \\
\hline Pachyramphus xanthogenys Salvadori \& Festa, 1898* NR & A & $E(1)$ & I \\
\hline Pachyramphus polychopterus (Vieillot, 1818) & A & $E(1)$ & I \\
\hline Pachyramphus marginatus (Lichtenstein, 1823) & $\mathrm{P} / \mathrm{B}$ & O & $\mathrm{R}$ \\
\hline Pachyramphus minor (Lesson, 1830) & $\mathrm{A}, \mathrm{P} / \mathrm{B}$ & G & I \\
\hline \multicolumn{4}{|l|}{ VIREONIDAE (7) } \\
\hline Cyclarhis gujanensis (Gmelin, 1789) & A & G & $\mathrm{F}$ \\
\hline Vireolanius leucotis (Swainson, 1838) & $\mathrm{P} / \mathrm{B}$ & G & I \\
\hline Vireo olivaceus (Linnaeus, 1766)* M & A & G & $\mathrm{R}$ \\
\hline Vireo flavoviridis (Cassin, 1851)* M & A & O & $\mathrm{R}$ \\
\hline
\end{tabular}


(Continua)

\begin{tabular}{|c|c|c|c|}
\hline Famílias e espécies & FIT & $\mathrm{D}$ & $A B$ \\
\hline Hylophilus thoracicus Temminck, 1822 & A & O & $\mathrm{I}$ \\
\hline Hylophilus hypoxanthus Pelzeln, 1868 & $\mathrm{~A}, \mathrm{P} / \mathrm{B}$ & O & I \\
\hline Hylophilus ochraceiceps Sclater, 1859 & $\mathrm{P} / \mathrm{B}$ & $E(2)$ & I \\
\hline \multicolumn{4}{|l|}{ CORVIDAE (1) } \\
\hline Cyanocorax violaceus Du Bus, 1847 & A & O & I \\
\hline \multicolumn{4}{|l|}{ HIRUNDINIDAE (5) } \\
\hline Atticora fasciata (Gmelin, 1789) & $\mathrm{R}$ & O & Co \\
\hline Stelgidopteryx ruficollis (Vieillot, 1817) & $\mathrm{R}$ & O & Co \\
\hline Progne tapera (Vieillot, 1817) & $\mathrm{R}$ & $E(1)$ & $\mathrm{F}$ \\
\hline Progne chalybea (Gmelin, 1789) & $\mathrm{R}$ & O & $\mathrm{F}$ \\
\hline Tachycineta albiventer (Boddaert, 1783) & $\mathrm{R}$ & O & $\mathrm{F}$ \\
\hline \multicolumn{4}{|l|}{ TROGLODYTIDAE (3) } \\
\hline Microcerculus marginatus (Sclater, 1855) & $\mathrm{P} / \mathrm{B}$ & $E(4)$ & I \\
\hline Campylorhynchus turdinus (Wied, 1831) & $\mathrm{A}, \mathrm{P} / \mathrm{B}$ & G & $\mathrm{F}$ \\
\hline Pheugopedius genibarbis (Swainson, 1838) & $\mathrm{A}, \mathrm{P} / \mathrm{B}$ & G & I \\
\hline \multicolumn{4}{|l|}{ POLIOPTILIDAE (2) } \\
\hline Ramphocaenus melanurus Vieillot, 1819 & A & O & $\mathrm{F}$ \\
\hline Polioptila plumbea (Gmelin, 1788)* DL & $\mathrm{P} / \mathrm{B}$ & O & $\mathrm{F}$ \\
\hline \multicolumn{4}{|l|}{ TURDIDAE (4) } \\
\hline Turdus nudigenis Lafresnaye, 1848 & A & O & I \\
\hline Turdus hauxwelli Lawrence, 1869 & A & O & $\mathrm{R}$ \\
\hline Turdus lawrencii Coues, 1880 & $A, P / B$ & O & $R$ \\
\hline Turdus ignobilis Sclater, 1858 & A & $E(1), G$ & $\mathrm{~F}$ \\
\hline \multicolumn{4}{|l|}{ COEREBIDAE (1) } \\
\hline Coereba flaveola (Linnaeus, 1758) & A & O & I \\
\hline \multicolumn{4}{|l|}{ THRAUPIDAE (19) } \\
\hline Saltator grossus (Linnaeus, 1766) & $\mathrm{P} / \mathrm{B}$ & G & $\mathrm{R}$ \\
\hline Saltator maximus (Statius Muller, 1776) & A & O & I \\
\hline Saltator coerulescens Vieillot, 1817 & A & O & I \\
\hline Cissopis leverianus (Gmelin, 1788) & A & O & $R$ \\
\hline Lamprospiza melanoleuca (Vieillot, 1817) & $\mathrm{P} / \mathrm{B}$ & $E(1), G$ & I \\
\hline Tachyphonus luctuosus d'Orbigny \& Lafresnaye, 1837 & $\mathrm{P} / \mathrm{B}$ & O & $\mathrm{R}$ \\
\hline Lanio versicolor (d'Orbigny \& Lafresnaye, 1837) & $\mathrm{P} / \mathrm{B}$ & G & I \\
\hline Ramphocelus carbo (Pallas, 1764) & A & O & I \\
\hline Ramphocelus nigrogularis (Spix, 1825) & A & O & $\mathrm{F}$ \\
\hline Thraupis episcopus (Linnaeus, 1766) & A & O & I \\
\hline Thraupis palmarum (Wied, 1823) & $\mathrm{A}, \mathrm{P} / \mathrm{B}$ & 0 & $\mathrm{R}$ \\
\hline
\end{tabular}


(Conclusão)

\begin{tabular}{|c|c|c|c|}
\hline Famílias e espécies & FIT & $\mathrm{D}$ & $A B$ \\
\hline Tangara chilensis (Vigors, 1832) & $\mathrm{A}, \mathrm{P} / \mathrm{B}$ & G & $\mathrm{I}$ \\
\hline Tangara schrankii (Spix, 1825) & $\mathrm{P} / \mathrm{B}$ & O & I \\
\hline Tangara gyrola (Linnaeus, 1758) & $\mathrm{P} / \mathrm{B}$ & G & I \\
\hline Tangara nigrocincta (Bonaparte, 1838) & A & O & $\mathrm{R}$ \\
\hline Tersina viridis (Illiger, 1811) & A & G & $\mathrm{F}$ \\
\hline Dacnis lineata (Gmelin, 1789) & $\mathrm{A}, \mathrm{P} / \mathrm{B}$ & O & I \\
\hline Dacnis cayana (Linnaeus, 1766) & $\mathrm{P} / \mathrm{B}$ & O & $\mathrm{R}$ \\
\hline Hemithraupis guira (Linnaeus, 1766) & $\mathrm{A}, \mathrm{P} / \mathrm{B}$ & $\mathrm{O}$ & $\mathrm{F}$ \\
\hline \multicolumn{4}{|l|}{ EMBERIZIDAE (5) } \\
\hline Ammodramus aurifrons (Spix, 1825) & $\mathrm{R}$ & O & $\mathrm{F}$ \\
\hline Sporophila caerulescens (Vieillot, 1823)* M & $\mathrm{R}$ & $\mathrm{O}$ & $\mathrm{R}$ \\
\hline Tiaris sp.* ED & A & G & $\mathrm{R}$ \\
\hline Arremon taciturnus (Hermann, 1783) & A & $E(2), G$ & $\mathrm{R}$ \\
\hline Paroaria gularis (Linnaeus, 1766) & A & $E(1)$ & $\mathrm{F}$ \\
\hline \multicolumn{4}{|l|}{ CARDINALIDAE (2) } \\
\hline Habia rubica (Vieillot, 1817) & $\mathrm{P} / \mathrm{B}$ & G & $\mathrm{F}$ \\
\hline Cyanoloxia cyanoides (Lafresnaye, 1847) & A & $E(4)$ & I \\
\hline \multicolumn{4}{|l|}{ PARULIDAE (1) } \\
\hline Phaeothlypis fulvicauda (Spix, 1825) & A & $E(1)$ & I \\
\hline \multicolumn{4}{|l|}{ ICTERIDAE (10) } \\
\hline Psarocolius angustifrons alfredi (Spix, 1824)* NR & A & $E(2)$ & $\mathrm{F}$ \\
\hline Psarocolius decumanus (Pallas, 1769) & $\mathrm{A}, \mathrm{P} / \mathrm{B}$ & $G$ & I \\
\hline Psarocolius bifasciatus (Spix, 1824) & $\mathrm{A}, \mathrm{P} / \mathrm{B}$ & G & I \\
\hline Procacicus solitarius (Vieillot, 1816) & A & $E(5), G$ & I \\
\hline Cacicus oseryi (Deville, 1849)* DL & $\mathrm{P} / \mathrm{B}$ & 0 & I \\
\hline Cacicus cela (Linnaeus, 1758) & $\mathrm{A}, \mathrm{P} / \mathrm{B}$ & $E(1), G$ & I \\
\hline Icterus cayanensis (Linnaeus, 1766) & A & G & $\mathrm{R}$ \\
\hline Icterus croconotus (Wagler, 1829) & A & $E(2)$ & I \\
\hline Molothrus oryzivorus (Gmelin, 1788) & A & O & $\mathrm{F}$ \\
\hline Molothrus bonariensis (Gmelin, 1789) & A & O & $\mathrm{R}$ \\
\hline \multicolumn{4}{|l|}{ FRINGILLIDAE (5) } \\
\hline Euphonia chlorotica (Linnaeus, 1766) & A & O & I \\
\hline Euphonia laniirostris d'Orbigny \& Lafresnaye, 1837 & A & O & $\mathrm{R}$ \\
\hline Euphonia chrysopasta Sclater \& Salvin, 1869 & $\mathrm{P} / \mathrm{B}$ & O & I \\
\hline Euphonia minuta Cabanis, 1849 & $\mathrm{P} / \mathrm{B}$ & G & I \\
\hline Euphonia rufiventris (Vieillot, 1819) & $\mathrm{A}, \mathrm{P} / \mathrm{B}$ & $\mathrm{O}$ & I \\
\hline
\end{tabular}


